MATHEMATICS OF COMPUTATION

Volume 71, Number 237, Pages 165-196

S 0025-5718(00)01300-4

Article electronically published on October 17, 2000

\title{
QUINCUNX FUNDAMENTAL REFINABLE FUNCTIONS AND QUINCUNX BIORTHOGONAL WAVELETS
}

\author{
BIN HAN AND RONG-QING JIA
}

\begin{abstract}
We analyze the approximation and smoothness properties of quincunx fundamental refinable functions. In particular, we provide a general way for the construction of quincunx interpolatory refinement masks associated with the quincunx lattice in $\mathbb{R}^{2}$. Their corresponding quincunx fundamental refinable functions attain the optimal approximation order and smoothness order. In addition, these examples are minimally supported with symmetry. For two special families of such quincunx interpolatory masks, we prove that their symbols are nonnegative. Finally, a general way of constructing quincunx biorthogonal wavelets is presented. Several examples of quincunx interpolatory masks and quincunx biorthogonal wavelets are explicitly computed.
\end{abstract}

\section{INTRODUCTION}

In this paper, we are interested in bivariate fundamental refinable functions with quincunx dilation matrices. A function $\phi$ is said to be fundamental if $\phi$ is continuous, $\phi(0)=1$ and $\phi(\beta)=0$ for all $\beta \in \mathbb{Z}^{s} \backslash\{0\}$. An $s \times s$ integer matrix $M$ is called a dilation matrix if $\lim _{n \rightarrow \infty} M^{-n}=0$, i.e., all the eigenvalues of a dilation matrix $M$ are greater than one in modulus. In this paper, we are particularly interested in the following two dilation matrices:

$$
Q=\left(\begin{array}{cc}
1 & -1 \\
1 & 1
\end{array}\right) \quad \text { and } \quad T=\left(\begin{array}{cc}
1 & 1 \\
1 & -1
\end{array}\right) .
$$

A refinable function $\phi$ satisfies the following refinement equation

$$
\phi=\sum_{\beta \in \mathbb{Z}^{s}} a(\beta) \phi(M \cdot-\beta)
$$

with a dilation matrix $M$, where $a$ is a finitely supported sequence on $\mathbb{Z}^{s}$ called the (refinement) mask. When $\sum_{\beta \in \mathbb{Z}^{s}} a(\beta)=|\operatorname{det} M|$, it is known that there exists a unique compactly supported distributional solution, denoted by $\phi_{a}^{M}$ and called the normalized solution, to the refinement equation (1.2) subject to the condition $\widehat{\phi}_{a}^{M}(0)=1$.

Received by the editor July 13, 1999 and, in revised form, April 20, 2000.

2000 Mathematics Subject Classification. Primary 42C40, 41A25, 41A63, 65D05, 65D17.

Key words and phrases. Fundamental refinable functions, biorthogonal wavelets, quincunx lattice, approximation order, smoothness, coset by coset (CBC) algorithm.

The research of the first author was supported by a postdoctoral fellowship and Grant G121210654 from NSERC Canada.

The research of the second author was partially supported by NSERC Canada under Grant OGP 121336.

(C)2000 American Mathematical Society 
If a compactly supported function $\phi$ is fundamental and satisfies the refinement equation (1.2) with a finitely supported refinement mask $a$ and a dilation matrix $M$, then it is necessary that

$$
a(0)=1 \quad \text { and } \quad a(\beta)=0 \quad \forall \beta \in M \mathbb{Z}^{s} \backslash\{0\} .
$$

A finitely supported sequence $a$ on $\mathbb{Z}^{s}$ is called an interpolatory (refinement) mask if it satisfies the above condition (1.3) with a dilation matrix $M$ and $\sum_{\beta \in \mathbb{Z}^{s}} a(\beta)=$ $|\operatorname{det} M|$.

In order to solve the refinement equation (1.2), we start with an initial function $\phi_{0}$ given by

$$
\phi_{0}\left(x_{1}, \cdots, x_{s}\right)=\prod_{j=1}^{s} \chi\left(x_{j}\right), \quad\left(x_{1}, \cdots, x_{s}\right) \in \mathbb{R}^{s},
$$

where $\chi$ is the hat function defined by $\chi(x):=\max \{1-|x|, 0\}, x \in \mathbb{R}$. Then we employ the iteration scheme $Q_{a}^{n} \phi_{0}, n=0,1,2, \cdots$, where $Q_{a}$ is the bounded linear operator on $L_{p}\left(\mathbb{R}^{s}\right)(1 \leq p \leq \infty)$ given by

$$
Q_{a} f:=\sum_{\beta \in \mathbb{Z}^{s}} a(\beta) f(M \cdot-\beta), \quad f \in L_{p}\left(\mathbb{R}^{s}\right) .
$$

This iteration scheme is called a subdivision scheme associated with the mask a and the dilation matrix $M$ (see [1]). If the mask is an interpolatory mask, this subdivision scheme is called an interpolatory subdivision scheme. We say that the subdivision scheme associated with a mask $a$ and a dilation matrix $M$ converges in the $L_{p}$ norm if the sequence of functions $Q_{a}^{n} \phi_{0}$ converges to a function $f \in L_{p}\left(\mathbb{R}^{s}\right)$ in the $L_{p}$ norm, i.e., $\lim _{n \rightarrow \infty}\left\|Q_{a}^{n} \phi_{0}-f\right\|_{p}=0$. If this is the case, then $Q_{a} f=f$ and $f=\phi_{a}^{M}$.

Let $\ell\left(\mathbb{Z}^{s}\right)$ denote the linear space of all sequences on $\mathbb{Z}^{s}$ and $\ell_{0}\left(\mathbb{Z}^{s}\right)$ denote the subspace of all finitely supported sequences on $\mathbb{Z}^{s}$. The difference operator $\nabla_{i}$ on $\ell_{0}\left(\mathbb{Z}^{s}\right)$ is defined as $\nabla_{i} \lambda=\lambda-\lambda\left(\cdot-e_{i}\right), \lambda \in \ell_{0}\left(\mathbb{Z}^{s}\right)$, where $e_{i}$ is the $i$-th coordinate unit vector in $\mathbb{R}^{s}$. By $\delta$ we denote the Dirac sequence given by $\delta(0)=1$ and $\delta(\beta)=0$ for all $\beta \in \mathbb{Z}^{s} \backslash\{0\}$. For any mask $a \in \ell_{0}\left(\mathbb{Z}^{s}\right)$ and a general dilation matrix $M$, it was demonstrated in [14 that the subdivision scheme associated with the mask $a$ and the dilation matrix $M$ converges in the $L_{p}$ norm if and only if

$$
\lim _{n \rightarrow \infty}\left\|\nabla_{i} S_{a, M}^{n} \delta\right\|_{p}^{1 / n}<m^{1 / p} \quad \forall i=1, \cdots, s,
$$

where $m:=|\operatorname{det} M|$ and the subdivision operator $S_{a, M}$ is defined by

$$
S_{a, M} \lambda(\alpha):=\sum_{\beta \in \mathbb{Z}^{s}} a(\alpha-M \beta) \lambda(\beta), \quad \alpha \in \mathbb{Z}^{s}, \lambda \in \ell\left(\mathbb{Z}^{s}\right) .
$$

Let $a$ be an interpolatory refinement mask with a dilation matrix $M$. Then the normalized solution $\phi_{a}^{M}$ of the refinement equation (1.2) with the mask $a$ and the dilation matrix $M$ is fundamental if and only if the subdivision scheme associated with the mask $a$ and the dilation matrix $M$ converges in the $L_{\infty}$ norm.

Let $Q$ and $T$ be the matrices defined in (1.1). Then

$$
Q \mathbb{Z}^{2}=T \mathbb{Z}^{2}=\left\{\left(\beta_{1}, \beta_{2}\right) \in \mathbb{Z}^{2}: \beta_{1}+\beta_{2} \text { is an even number }\right\} .
$$


The lattice $Q \mathbb{Z}^{2}$ is called the quincunx lattice. Thus, we say that a sequence $a$ on $\mathbb{Z}^{2}$ is a quincunx interpolatory mask if $\sum_{\beta \in \mathbb{Z}^{2}} a(\beta)=2$ and

$$
a(0)=1 \quad \text { and } \quad a(\beta)=0 \quad \forall \beta \in Q \mathbb{Z}^{2} \backslash\{0\} .
$$

Let $a$ be a quincunx interpolatory mask. If the normalized solution $\phi_{a}^{Q}$ (or $\phi_{a}^{T}$ ) to the refinement equation (1.2) is fundamental, then it is called a quincunx fundamental refinable function.

Interpolatory subdivision schemes play an important role in computer graphics and wavelet analysis. See [9] for their applications to computer aided geometric design, and see [4] for their applications to wavelet decompositions.

In the current literature for the univariate case, Deslauriers and Dubuc in [7] proposed a general method to construct symmetric interpolatory subdivision schemes. For the multivariate case, Dyn, Gregory and Levin [10] constructed the so-called butterfly scheme which is a $C^{1}$ bivariate interpolatory subdivision scheme, while Deslauriers, Dubois and Dubuc [8] obtained several continuous bivariate refinable and fundamental functions. Mongeau and Deslauriers [24] obtained several $C^{1}$ bivariate refinable and fundamental functions. Using convolutions of box splines with refinable distributions, Riemenschneider and Shen [25] constructed a family of bivariate interpolatory subdivision schemes with symmetry. Han and Jia [15] constructed a family of bivariate optimal interpolatory subdivision schemes with many desired properties.

However, all the above constructions in the multivariate case have used the dilation matrix $2 I_{2}$ only. Owing to some special properties of the matrices $Q$ and $T$, such as $|\operatorname{det} Q|=|\operatorname{det} T|=2, T^{2}=2 I_{2}$ and $Q^{4}=-4 I_{2}$, it is desirable to consider quincunx fundamental refinable functions and quincunx biorthogonal wavelets, i.e., biorthogonal wavelets with the dilation matrix $Q$ or $T$. See Cohen and Daubechies [4] for discussions on quincunx biorthogonal wavelets. Also, quincunx fundamental refinable functions automatically provide a family of primal refinable functions from which quincunx biorthogonal wavelets can be constructed. Quincunx biorthogonal wavelets are useful in image processing [21] because of their special properties. For biorthogonal wavelets, the reader is referred to $2,3,4,5,6,12,13,16,21,22,26,27$. and references therein.

The main purpose of this paper is to investigate and construct quincunx interpolatory masks and quincunx biorthogonal wavelets with some desired properties.

The structure of this paper is as follows. In Section 2, we shall investigate the optimal approximation order and smoothness order of quincunx fundamental refinable functions with respect to their support. In Section 3, we shall propose a family of quincunx interpolatory masks such that they are minimally supported and have symmetry. Their associated quincunx fundamental refinable functions have optimal approximation order and smoothness order. In particular, for two special families of such quincunx interpolatory masks, we prove that their symbols are nonnegative. In Section 4, several examples of quincunx interpolatory masks are explicitly computed. Both the $L_{2}$ and the $L_{\infty}$ smoothness described by the critical exponents of their quincunx fundamental refinable functions are calculated. Finally, in Section 5, we discuss how to construct quincunx biorthogonal wavelets by using the coset by coset $(\mathrm{CBC})$ algorithm proposed in [13] and [2]. Examples are provided to illustrate the general theory. 


\section{ApPROXIMATION ORDER AND SMOOTHNESS ORDER}

In this section we shall investigate the approximation and smoothness properties of quincunx fundamental refinable functions.

For a compactly supported function $\phi$ in $L_{p}\left(\mathbb{R}^{s}\right)(1 \leq p \leq \infty)$, we define

$$
S(\phi):=\left\{\sum_{\alpha \in \mathbb{Z}^{s}} \phi(\cdot-\alpha) \lambda(\alpha): \lambda \in \ell\left(\mathbb{Z}^{s}\right)\right\} .
$$

For $h>0, S^{h}$ is defined by $S^{h}:=\{g(\cdot / h): g \in S(\phi)\}$. For a positive integer $k$, we say that $S(\phi)$ provides approximation order $k$ if, for each sufficiently smooth function $f$ in $L_{p}\left(\mathbb{R}^{s}\right)$, there exists a positive constant $C$ such that

$$
\inf _{g \in S^{h}}\|f-g\|_{p} \leq C h^{k} \quad \forall h>0 .
$$

The concept of stability plays an important role in wavelet analysis. Let $\phi$ be a compactly supported function in $L_{p}\left(\mathbb{R}^{s}\right)(1 \leq p \leq \infty)$. We say that the shifts of $\phi$ are stable if there are two positive constants $C_{1}$ and $C_{2}$ such that

$$
C_{1}\|\lambda\|_{p} \leq\left\|\sum_{\alpha \in \mathbb{Z}^{s}} \lambda(\alpha) \phi(\cdot-\alpha)\right\|_{p} \leq C_{2}\|\lambda\|_{p} \quad \forall \lambda \in \ell_{0}\left(\mathbb{Z}^{s}\right) .
$$

Let $a$ be a sequence on $\mathbb{Z}^{s}$. For a positive integer $k$, we say that $a$ satisfies the sum rules of order $k$ with a dilation matrix $M$ if

$$
\sum_{\beta \in M \mathbb{Z}^{s}} a(\varepsilon+\beta) p(\varepsilon+\beta)=\sum_{\beta \in M \mathbb{Z}^{s}} a(\beta) p(\beta) \quad \forall \varepsilon \in \mathbb{Z}^{s}, p \in \Pi_{k-1},
$$

where $\Pi_{k-1}$ denotes the set of all polynomials of (total) degree at most $k-1$. Note that (2.2) depends only on the lattice $M \mathbb{Z}^{s}$. If a mask $a$ on $\mathbb{Z}^{2}$ satisfies (2.2) with the quincunx lattice $Q \mathbb{Z}^{2}$, then we say that $a$ satisfies the sum rules of order $k$ with respect to the quincunx lattice.

Now suppose $\phi$ is the normalized solution of the refinement equation (1.2) with a mask $a$ and a dilation matrix $M$. It was proved by Jia in [17] that if the shifts of $\phi$ are stable, then $S(\phi)$ provides approximation order $k$ if and only if the mask $a$ satisfies the sum rules of order $k$. Note that a fundamental function has stable shifts. Thus, in particular, if $\phi$ is a fundamental refinable function with a mask $a$ and a dilation matrix $M$, then $S(\phi)$ provides approximation order $k$ if and only if $a$ satisfies the sum rules of order $k$ with the dilation matrix $M$.

Deslauriers and Dubuc in [7] proposed a family of interpolatory masks $b_{r}(r \in \mathbb{N})$ with the dilation matrix $M=(2)$. Their construction was restated in [15] as follows.

Theorem 2.1. Let the dilation matrix $M=(2)$ and $a \in \ell_{0}(\mathbb{Z})$ be an interpolatory refinement mask on $\mathbb{Z}$ satisfying the sum rules of order $k$. If a is supported on an interval $[1-2 r, 2 r-1]$ for some $r \in \mathbb{N}$, then $k \leq 2 r$. Moreover, there exists a unique interpolatory refinement mask, denoted by $b_{r}$, such that it is supported on $[1-2 r, 2 r-1]$ and satisfies the sum rules of order $2 r$. In fact, an explicit formula for $b_{r}$ is

$$
b_{r}(2 j-1):=(-1)^{j+1} \frac{\prod_{k=1}^{r}(2 k-1)^{2}}{2^{2 r-1}(2 j-1) \cdot(r-1+j) !(r-j) !}, \quad 1-r \leq j \leq r .
$$

Now we have the following result for quincunx interpolatory masks. 
Theorem 2.2. Let a be a quincunx interpolatory mask supported on

$$
\left\{\left(\beta_{1}, \beta_{2}\right) \in \mathbb{Z}^{2}:\left|\beta_{1}\right|+\left|\beta_{2}\right|<2 r\right\},
$$

where $r$ is a positive integer. If a satisfies the sum rules of order $k$, then $k \leq 2 r$.

Proof. Let $b$ be the sequence on $\mathbb{Z}$ defined by

$$
b(i):=\sum_{j \in \mathbb{Z}} a(i-j, j), \quad i \in \mathbb{Z} .
$$

Since $a$ is a quincunx interpolatory mask, it is easily seen that $b$ is an interpolatory mask with the dilation matrix $M=(2)$. Since $a$ satisfies the sum rules of order $k$, it follows that

$$
\begin{aligned}
\sum_{i \in \mathbb{Z}} b & (2 i+1)(2 i+1)^{m} \\
& =\sum_{i \in \mathbb{Z}} \sum_{j \in \mathbb{Z}} a(2 i+1-j, j)(2 i+1-j+j)^{m}=\delta(m) \quad \forall 0 \leq m<k .
\end{aligned}
$$

Therefore, $b$ satisfies the sum rules of order at least $k$. Since $b$ is an interpolatory mask supported on $[1-2 r, 2 r-1]$, by Theorem 2.1 we have $k \leq 2 r$. This completes the proof.

In the rest of this section, we shall study the smoothness property of quincunx fundamental refinable functions.

For $0<\eta \leq 1$, the Lipschitz space $\operatorname{Lip}\left(\eta, L_{p}\left(\mathbb{R}^{s}\right)\right)$ consists of those functions $f$ in $L_{p}\left(\mathbb{R}^{s}\right)$ for which

$$
\|f-f(\cdot-t)\|_{p} \leq C\|t\|^{\eta} \quad \forall t \in \mathbb{R}^{s},
$$

where the constant $C$ depends only on $f$. Let $\mathbb{Z}_{+}^{s}:=\left\{\left(\beta_{1}, \cdots, \beta_{s}\right) \in \mathbb{Z}^{s}: \beta_{i} \geq\right.$ $0 \forall i=1, \cdots, s\}$. The $L_{p}$ smoothness of a function $f \in L_{p}\left(\mathbb{R}^{s}\right)$ is described by its $L_{p}$ critical exponent $\nu_{p}(f)$ defined by

$$
\nu_{p}(f):=\sup \left\{n+\eta \quad: \quad \frac{\partial^{\mu} f}{\partial x^{\mu}} \in \operatorname{Lip}\left(\eta, L_{p}\left(\mathbb{R}^{s}\right)\right) \quad \forall \mu \in \mathbb{Z}_{+}^{s},|\mu|=n\right\} .
$$

In [18, Jia completely characterized the $L_{2}$ critical exponent of a refinable function with an isotropic dilation matrix in terms of its mask provided that the shifts of the refinable function are stable. The following result is a straightforward generalization of Theorem 3.5 in Han 13 .

Theorem 2.3. Let $\phi$ be the normalized solution of the refinement equation (1.2) with a finitely supported mask $a \in \ell_{0}\left(\mathbb{Z}^{s}\right)$ and an $s \times s$ dilation matrix $M$ such that $\sum_{\beta \in \mathbb{Z}^{s}} a(\beta)=m:=|\operatorname{det} M|$. Suppose that $M^{j}$ is a multiple of the identity matrix for some positive integer $j$. For any nonnegative integer $k$, let

$$
\sigma_{k, p}^{M}(a):=\max \left\{\lim _{n \rightarrow \infty}\left\|\nabla_{i}^{k} S_{a, M}^{n} \delta\right\|_{p}^{1 / n}: i=1, \cdots, s\right\},
$$

where the subdivision operator $S_{a, M}$ is defined in (1.4). Then

$$
\min \left\{k, \nu_{p}(\phi)\right\} \geq s / p-s \log _{m} \sigma_{k, p}^{M}(a) .
$$

In addition, if the shifts of $\phi$ are stable, then

$$
\min \left\{k, \nu_{p}(\phi)\right\}=s / p-s \log _{m} \sigma_{k, p}^{M}(a) .
$$


In [14, it was demonstrated that $\sigma_{k, 2}^{M}(a)$ can be computed by calculating the spectral radius of a certain finite matrix. Let $b$ be the sequence given by

$$
b(\alpha):=\sum_{\beta \in \mathbb{Z}^{s}} a(\alpha+\beta) \overline{a(\beta)}, \quad \alpha \in \mathbb{Z}^{s} .
$$

The transition operator $T_{b, M}$ associated with the sequence $b$ and the dilation matrix $M$ is defined by

$$
T_{b, M} \lambda(\alpha)=\sum_{\beta \in \mathbb{Z}^{s}} b(M \alpha-\beta) \lambda(\beta), \quad \alpha \in \mathbb{Z}^{s}, \lambda \in \ell_{0}\left(\mathbb{Z}^{s}\right) .
$$

From Theorem 4.1 in [14, we have

$$
\sigma_{k, 2}^{M}(a)=\sqrt{\rho\left(\left.T_{b, M}\right|_{W}\right)}
$$

where $\rho\left(\left.T_{b, M}\right|_{W}\right)$ is the spectral radius of the operator $T_{b, M}$ restricted to the finite dimensional space $W$, and $W$ is the minimal invariant subspace of $T_{b, M}$ generated by $\Delta_{j}^{k} \delta, j=1, \cdots, s$, where

$$
\Delta_{j} \lambda(\alpha):=-\lambda\left(\alpha-e_{j}\right)+2 \lambda(\alpha)-\lambda\left(\alpha+e_{j}\right), \quad \alpha \in \mathbb{Z}^{s}, \lambda \in \ell\left(\mathbb{Z}^{s}\right) .
$$

The symbol of a sequence $a$ on $\mathbb{Z}^{s}$ is defined by

$$
\widetilde{a}(z):=\sum_{\beta \in \mathbb{Z}^{s}} a(\beta) z^{\beta}, \quad z \in(\mathbb{C} \backslash\{0\})^{s} .
$$

We say that the symbol of a mask $a$ is nonnegative if $\widetilde{a}\left(e^{-i \xi}\right) \geq 0$ for all $\xi \in \mathbb{R}^{s}$. Let $a$ be a finitely supported mask on $\mathbb{Z}^{s}$ with a nonnegative symbol. Then from Theorem 4.1 in 14, we have

$$
\sigma_{2 k, \infty}^{M}(a)=\rho\left(\left.T_{a, M}\right|_{W}\right),
$$

where the finite dimensional space $W$ is the minimal invariant subspace of $T_{a, M}$ generated by $\Delta_{j}^{k} \delta, j=1, \cdots, s$. For discussion on subdivision operators and transition operators, the reader is referred to [11, 17, 20].

Based on Theorem 2.3 we have the following result:

Theorem 2.4. Let $\phi_{a}^{T}$ be a fundamental refinable function with a finitely supported mask $a$ and the dilation matrix $T$ defined in (1.1). Suppose $a$ is supported on

$$
\left\{\left(\beta_{1}, \beta_{2}\right) \in \mathbb{Z}^{2}:\left|\beta_{1}\right|+\left|\beta_{2}\right|<2 r,\left|\beta_{2}\right|<r\right\},
$$

where $r$ is a positive integer. If a satisfies the sum rules of order $2 r$, then

$$
\nu_{p}\left(\phi_{a}^{T}\right) \leq \nu_{p}\left(\phi_{b_{r}}\right) \quad \forall 1 \leq p \leq \infty,
$$

where $\phi_{b_{r}}$ is the fundamental refinable function with the mask $b_{r}$ given in Theorem 2.1.

Proof. Define a sequence $b$ on $\mathbb{Z}$ as follows:

$$
b(j)=\sum_{i \in \mathbb{Z}} a(i, j) / 2, \quad j \in \mathbb{Z} .
$$

We claim that $b(j)=\delta(j)$ for all $j \in \mathbb{Z}$. Since $a$ is a quincunx interpolatory mask and $a$ satisfies the sum rules of order $2 r$, we have

$$
\sum_{j \in \mathbb{Z}} b(j) j^{m}=\sum_{j \in \mathbb{Z}} \sum_{i \in \mathbb{Z}} a(i, j) j^{m} / 2=\delta(m) \quad \forall 0 \leq m<2 r .
$$


Since $a$ is supported on $\left\{\left(\beta_{1}, \beta_{2}\right) \in \mathbb{Z}^{2}:\left|\beta_{1}\right|+\left|\beta_{2}\right|<2 r,\left|\beta_{2}\right|<r\right\}, b$ is supported on $[1-r, r-1]$. Therefore,

$$
\sum_{j=1-r}^{r-1} b(j) j^{m}=\delta(m) \quad \forall 0 \leq m<2 r .
$$

Since the coefficient matrix of the above linear system of equations is a Vandermonde matrix, it is easily seen that the unique solution is $b(j)=\delta(j)$ for all $j \in \mathbb{Z}$.

Let $c$ be the sequence on $\mathbb{Z}$ given by

$$
c(i):=\sum_{j \in \mathbb{Z}} S_{a, T}^{2} \delta(i, j) / 2, \quad i \in \mathbb{Z} .
$$

Then by the definition of the subdivision operator $S_{a, T}$, we have

$$
\begin{aligned}
c(i) & =\sum_{j \in \mathbb{Z}} S_{a, T} a(i, j) / 2=\sum_{j \in \mathbb{Z}} \sum_{\beta_{1} \in \mathbb{Z}} \sum_{\beta_{2} \in \mathbb{Z}} a\left(i-\beta_{1}-\beta_{2}, j-\beta_{1}+\beta_{2}\right) a\left(\beta_{1}, \beta_{2}\right) / 2 \\
& =\sum_{\beta_{1} \in \mathbb{Z}} \sum_{\beta_{2} \in \mathbb{Z}} a\left(\beta_{1}, \beta_{2}\right) \sum_{j \in \mathbb{Z}} a\left(i-\beta_{1}-\beta_{2}, j\right) / 2=\sum_{\beta_{1} \in \mathbb{Z}} \sum_{\beta_{2} \in \mathbb{Z}} a\left(\beta_{1}, \beta_{2}\right) \delta\left(i-\beta_{1}-\beta_{2}\right) \\
& =\sum_{\beta_{2} \in \mathbb{Z}} a\left(i-\beta_{2}, \beta_{2}\right),
\end{aligned}
$$

where we have used the fact that $\sum_{j \in \mathbb{Z}} a\left(i-\beta_{1}-\beta_{2}, j\right) / 2=b\left(i-\beta_{1}-\beta_{2}\right)=$ $\delta\left(i-\beta_{1}-\beta_{2}\right)$. From the proof of Theorem 2.2 , we see that the sequence $c$ must be equal to the sequence $b_{r}$ since $a$ satisfies the sum rules of order $2 r$. Observe that

$$
\phi_{a}^{T}=\sum_{\beta \in \mathbb{Z}^{2}} S_{a, T}^{2} \delta(\beta) \phi_{a}^{T}(2 \cdot-\beta) .
$$

Let $a_{2}:=S_{a, T}^{2} \delta$. Since $a$ is finitely supported, the sequence $a_{2}$ is supported on $[-N, N]^{2}$ for some positive integer $N$. Note that $c(i)=\sum_{j \in \mathbb{Z}} a_{2}(i, j) / 2$. By induction, it is easily seen that

$$
S_{b_{r},(2)}^{n} \delta(i)=S_{c,(2)}^{n} \delta(i)=2^{-n} \sum_{j \in \mathbb{Z}} S_{a_{2}, 2 I_{2}}^{n} \delta(i, j), \quad i \in \mathbb{Z}, n \in \mathbb{N}
$$

and $S_{a_{2}, 2 I_{2}}^{n} \delta$ is supported on $\left[-2^{n} N, 2^{n} N\right]^{2}$. Therefore, for any positive integer $k$,

$$
\nabla_{1}^{k} S_{b_{r},(2)}^{n} \delta(i)=2^{-n} \sum_{j=-2^{n} N}^{2^{n} N} \nabla_{1}^{k} S_{a_{2}, 2 I_{2}}^{n} \delta(i, j) .
$$

Applying the Hölder inequality to the above equality, we have

$$
\begin{aligned}
\left|\nabla_{1}^{k} S_{b_{r},(2)}^{n} \delta(i)\right|^{p} & \leq 2^{-n p}\left(2^{n+1} N+1\right)^{p / q} \sum_{j \in \mathbb{Z}}\left|\nabla_{1}^{k} S_{a_{2}, 2 I_{2}}^{n} \delta(i, j)\right|^{p} \\
& \leq 2^{-n} C_{1} \sum_{j \in \mathbb{Z}}\left|\nabla_{1}^{k} S_{a_{2}, 2 I_{2}}^{n} \delta(i, j)\right|^{p}
\end{aligned}
$$

where $1 / p+1 / q=1$ and $C_{1}=(2 N+1)^{p / q}$. Therefore,

$$
\left\|\nabla_{1}^{k} S_{b_{r},(2)}^{n} \delta\right\|_{p} \leq C_{1}^{1 / p} 2^{-n / p}\left\|\nabla_{1}^{k} S_{a_{2}, 2 I_{2}}^{n} \delta\right\|_{p}
$$

from which it follows that

$$
\sigma_{k, p}^{2 I_{2}}\left(a_{2}\right) \geq \lim _{n \rightarrow \infty}\left\|\nabla_{1}^{k} S_{a_{2}, 2 I_{2}}^{n} \delta\right\|_{p}^{1 / n} \geq 2^{1 / p} \lim _{n \rightarrow \infty}\left\|\nabla_{1}^{k} S_{b_{r},(2)}^{n} \delta\right\|_{p}^{1 / n}=2^{1 / p} \sigma_{k, p}^{(2)}\left(b_{r}\right) .
$$


On the other hand, since $\phi_{a}^{T}$ is a fundamental function, the shifts of $\phi_{a}^{T}$ are stable. For a sufficiently large integer $k$, by Theorem [2.3,

$$
\begin{aligned}
\nu_{p}\left(\phi_{a}^{T}\right)=2 / p-\log _{2} \sigma_{k, p}^{2 I_{2}}\left(a_{2}\right) & \leq 2 / p-\log \left(2^{1 / p} \sigma_{k, p}^{(2)}\left(b_{r}\right)\right) \\
& =1 / p-\log _{2} \sigma_{k, p}^{(2)}\left(b_{r}\right)=\nu_{p}\left(\phi_{b_{r}}\right) .
\end{aligned}
$$

We are done.

\section{Construction of QuincunX interpolatory MASKS}

Our construction of quincunx interpolatory masks relies on the solvability of certain linear systems of equations. By $\mathbb{Z}_{+}^{2}$ we denote the set of all elements $\mu=$ $\left(\mu_{1}, \mu_{2}\right) \in \mathbb{Z}^{2}$ with both $\mu_{1}$ and $\mu_{2}$ nonnegative. Let $|\mu|$ denote $\left|\mu_{1}\right|+\left|\mu_{2}\right|$. For a positive integer $r \in \mathbb{N}$ and a nonnegative integer $k$, define

$$
\begin{aligned}
& \Gamma_{r}^{k}:=\left\{\left(\mu_{1}, \mu_{2}\right) \in \mathbb{Z}_{+}^{2}:\right. \mu_{1}+\mu_{2}<2 r+2 k, \\
&\left.\mu_{2}<2 r-1\right\} \backslash\{(0,2 j-1): j=1, \cdots, r-1\} .
\end{aligned}
$$

The cardinality of a set $E$ is denoted by $\# E$. To facilitate our discussion, we recall Lemma 4.2 in [15].

Lemma 3.1. Let $r$ be a positive integer and let $\Gamma_{r}^{0}$ be the set defined in (3.1). Let $p$ be a linear combination of the monomials $x_{1}^{\mu_{1}} x_{2}^{\mu_{2}},\left(\mu_{1}, \mu_{2}\right) \in \Gamma_{r}^{0}$. Let $L_{j}$ and $H_{j}(j=1, \cdots, r)$ be the lines $x_{1}-l_{j}=0$ and $x_{1}-h_{j}=0$, respectively, where $l_{1}, \cdots, l_{r}, h_{1}, \cdots, h_{r}$ are mutually distinct nonzero real numbers. Suppose $E$ is a subset of the union of these lines such that $\#\left(E \cap L_{j}\right)=\#\left(E \cap H_{j}\right)=2 j-1$ for each $j=1, \cdots, r$. If $p$ vanishes on $E$, then $p$ vanishes everywhere. Consequently, the square matrix $\left(t_{1}^{\mu_{1}} t_{2}^{\mu_{2}}\right)_{\left(t_{1}, t_{2}\right) \in E,\left(\mu_{1}, \mu_{2}\right) \in \Gamma_{r}^{0}}$ is nonsingular.

The following result is an extension of the above lemma.

Lemma 3.2. Let $r$ be a positive integer and $k$ a nonnegative integer. Let $p$ be a linear combination of the monomials $x_{1}^{\mu_{1}} x_{2}^{\mu_{2}},\left(\mu_{1}, \mu_{2}\right) \in \Gamma_{r}^{k}$ where $\Gamma_{r}^{k}$ is the set given in (3.1). Let $L_{j}$ and $H_{j}(j=1, \cdots, r+k)$ be the lines $x_{1}-l_{j}=0$ and $x_{1}-h_{j}=0$, respectively, where $l_{1}, \cdots, l_{r+k}, h_{1}, \cdots, h_{r+k}$ are mutually distinct nonzero real numbers. Suppose $E$ is a subset of the union of these lines such that $\#\left(E \cap L_{j}\right)=\#\left(E \cap H_{j}\right)=2 j-1$ for each $j=1, \cdots, r$ and $\#\left(E \cap L_{j}\right)=\#\left(E \cap H_{j}\right)=$ $2 r-1$ for each $j=r+1, \cdots, r+k$. If $p$ vanishes on $E$, then $p$ vanishes everywhere. Consequently, the square matrix $\left(t_{1}^{\mu_{1}} t_{2}^{\mu_{2}}\right)_{\left(t_{1}, t_{2}\right) \in E,\left(\mu_{1}, \mu_{2}\right) \in \Gamma_{r}^{k}}$ is nonsingular.

Proof. The proof proceeds by induction on $k$. The case $k=0$ follows from Lemma 3.1. Suppose $k \geq 1$ and the conclusion in Lemma 3.2 is true for $k-1$. We demonstrate that it is also true for $k$.

Since $p$ is a linear combination of the monomials $x_{1}^{\mu_{1}} x_{2}^{\mu_{2}},\left(\mu_{1}, \mu_{2}\right) \in \Gamma_{r}^{k}$, from the definition of $\Gamma_{r}^{k}$ we see that the degree of the univariate polynomial $p\left(l_{r+k}, x_{2}\right)$ is at most $2 r-2$. But $p\left(l_{r+k}, x_{2}\right)$ has $2 r-1$ zeros on $E \cap L_{r+k}$. Therefore, $p\left(l_{r+k}, x_{2}\right)=0$ for all $x_{2} \in \mathbb{R}$. It follows that $p\left(x_{1}, x_{2}\right)=\left(x_{1}-l_{r+k}\right) u\left(x_{1}, x_{2}\right)$, where $u$ is a polynomial in $x_{1}$ and $x_{2}$. It is easy to see that the degree of the univariate polynomial $u\left(h_{r+k}, x_{2}\right)$ is at most $2 r-2$. But $u\left(h_{r+k}, x_{2}\right)$ has $2 r-1$ zeros on $E \cap H_{r+k}$ since $h_{r+k} \neq l_{r+k}$. Therefore, $u\left(x_{1}, x_{2}\right)=\left(x_{1}-h_{r+k}\right) q\left(x_{1}, x_{2}\right)$. Thus, $p\left(x_{1}, x_{2}\right)=\left(x_{1}-l_{r+k}\right)\left(x_{1}-h_{r+k}\right) q\left(x_{1}, x_{2}\right)$. Since $l_{r+k} h_{r+k} \neq 0$, we observe that $q$ is a linear combination of the monomials $x_{1}^{\mu_{1}} x_{2}^{\mu_{2}},\left(\mu_{1}, \mu_{2}\right) \in \Gamma_{r}^{k-1}$. Moreover, $q$ vanishes on the set

$$
E^{\prime}:=\left\{E \cap L_{j}: j=1, \cdots, r+k-1\right\} \cup\left\{E \cap H_{j}: j=1, \cdots, r+k-1\right\} .
$$


By induction hypothesis, $q$ vanishes everywhere. Therefore, the polynomial $p$ vanishes everywhere.

Note that $\# E=\# \Gamma_{r}^{k}=2 r^{2}+(4 r-2) k$. In order to prove that the matrix $\left(t_{1}^{\mu_{1}} t_{2}^{\mu_{2}}\right)_{\left(t_{1}, t_{2}\right) \in E,\left(\mu_{1}, \mu_{2}\right) \in \Gamma_{r}^{k}}$ is nonsingular, it suffices to show that the linear system of homogeneous equations

$$
\sum_{\left(\mu_{1}, \mu_{2}\right) \in \Gamma_{r}^{k}} c_{\mu_{1}, \mu_{2}} t_{1}^{\mu_{1}} t_{2}^{\mu_{2}}=0, \quad\left(t_{1}, t_{2}\right) \in E,
$$

only has the trivial solution for $c_{\mu_{1}, \mu_{2}},\left(\mu_{1}, \mu_{2}\right) \in \Gamma_{r}^{k}$. For this purpose, let

$$
p\left(x_{1}, x_{2}\right):=\sum_{\left(\mu_{1}, \mu_{2}\right) \in \Gamma_{r}^{k}} c_{\mu_{1}, \mu_{2}} x_{1}^{\mu_{1}} x_{2}^{\mu_{2}} .
$$

Then $p\left(x_{1}, x_{2}\right)$ is a linear combination of the monomials $x_{1}^{\mu_{1}} x_{2}^{\mu_{2}},\left(\mu_{1}, \mu_{2}\right) \in \Gamma_{r}^{k}$ and it vanishes on $E$. By what has been proved, $p=0$. This completes the proof.

We are in a position to construct a family of quincunx interpolatory masks.

Theorem 3.3. Given a pair of nonnegative integers $m$ and $n$ with $m+n$ being an odd integer, there exists a unique quincunx interpolatory mask $q_{m, n}$ such that $q_{m, n}$ is supported on

$$
\left\{\left(\beta_{1}, \beta_{2}\right) \in \mathbb{Z}^{2}:\left|\beta_{1}\right| \leq m,\left|\beta_{2}\right| \leq n\right\},
$$

and $q_{m, n}$ satisfies the sum rules of order $m+n+1$ with respect to the quincunx lattice which is defined to be $\left\{\left(\beta_{1}, \beta_{2}\right) \in \mathbb{Z}^{2}: \beta_{1}+\beta_{2}\right.$ is an even integer $\}$.

Proof. Without loss of generality, we assume $n \leq m$. Define

$$
G_{m, n}:=\left\{\left(\beta_{1}, \beta_{2}\right) \in \mathbb{Z}^{2}:\left|\beta_{1}\right| \leq m,\left|\beta_{2}\right| \leq n, \beta_{1}+\beta_{2} \text { is an odd integer }\right\} .
$$

Let $\Gamma_{m, n}:=\Gamma_{n+1}^{\frac{m-n-1}{2}}$. Note that $\# \Gamma_{m, n}=\# G_{m, n}=2 m n+m+n+1$. We wish to prove that the square matrix $\left(\left(\beta_{1}+\beta_{2}\right)^{\mu_{1}}\left(\beta_{1}-\beta_{2}\right)^{\mu_{2}}\right)_{\left(\beta_{1}, \beta_{2}\right) \in G_{m, n},\left(\mu_{1}, \mu_{2}\right) \in \Gamma_{m, n}}$ is nonsingular. For this purpose, let $E_{m, n}:=\left\{\left(\beta_{1}+\beta_{2}, \beta_{1}-\beta_{2}\right):\left(\beta_{1}, \beta_{2}\right) \in G_{m, n}\right\}$.

We observe that $E_{m, n}$ intersects the line $x_{1} \pm(m+n+2-2 j)=0$ at exactly $2 j-1$ distinct points for $j=1, \cdots, n+1$ and it intersects the line $x_{1} \pm(2 j-2 n-3)=0$ at exactly $2 n+1$ distinct points for $j=n+2, \cdots, \frac{m+n+1}{2}$. Thus, Lemma 3.2 is applicable and we conclude that the square matrix

$$
\left(\left(\beta_{1}+\beta_{2}\right)^{\mu_{1}}\left(\beta_{1}-\beta_{2}\right)^{\mu_{2}}\right)_{\left(\beta_{1}, \beta_{2}\right) \in G_{m, n},\left(\mu_{1}, \mu_{2}\right) \in \Gamma_{m, n}}
$$

is nonsingular. Consequently, the linear system of equations

$$
\sum_{\left(\beta_{1}, \beta_{2}\right) \in G_{m, n}} c_{\beta_{1}, \beta_{2}}\left(\beta_{1}+\beta_{2}\right)^{\mu_{1}}\left(\beta_{1}-\beta_{2}\right)^{\mu_{2}}=\delta\left(\mu_{1}, \mu_{2}\right) \quad \forall\left(\mu_{1}, \mu_{2}\right) \in \Gamma_{m, n}
$$

has a unique solution for $\left\{c_{\beta_{1}, \beta_{2}}:\left(\beta_{1}, \beta_{2}\right) \in G_{m, n}\right\}$.

Let $c_{\beta_{1}, \beta_{2}},\left(\beta_{1}, \beta_{2}\right) \in G_{m, n}$ be the unique solution to the linear system (3.3). We claim that

$$
\sum_{\left(\beta_{1}, \beta_{2}\right) \in G_{m, n}} c_{\beta_{1}, \beta_{2}}\left(\beta_{1}+\beta_{2}\right)^{\mu_{1}}\left(\beta_{1}-\beta_{2}\right)^{\mu_{2}}=\delta\left(\mu_{1}, \mu_{2}\right)
$$

is valid for all $\left(\mu_{1}, \mu_{2}\right) \in \mathbb{Z}_{+}^{2}$ satisfying $\mu_{1}+\mu_{2}<m+n+1$. 
Observe that the set $G_{m, n}$ is symmetric about the origin. Therefore,

$$
\sum_{\left(\beta_{1}, \beta_{2}\right) \in G_{m, n}} c_{-\beta_{1},-\beta_{2}}\left(\beta_{1}+\beta_{2}\right)^{\mu_{1}}\left(\beta_{1}-\beta_{2}\right)^{\mu_{2}}=\delta\left(\mu_{1}, \mu_{2}\right) \quad \forall\left(\mu_{1}, \mu_{2}\right) \in \Gamma_{m, n} .
$$

By the uniqueness of the above system, we have $c_{-\beta_{1},-\beta_{2}}=c_{\beta_{1}, \beta_{2}}$ for all $\left(\beta_{1}, \beta_{2}\right) \in$ $G_{m, n}$. Thus, this symmetry of $c_{\beta_{1}, \beta_{2}}$ implies

$$
\sum_{\left(\beta_{1}, \beta_{2}\right) \in G_{m, n}} c_{\beta_{1}, \beta_{2}}\left(\beta_{1}-\beta_{2}\right)^{2 j-1}=0 \quad \forall j \in \mathbb{N} .
$$

Hence, (3.4) holds true for any $\left(\mu_{1}, \mu_{2}\right) \in \mathbb{Z}_{+}^{2}$ satisfying $\mu_{1}+\mu_{2}<m+n+1$ and $\mu_{2}<2 n+1$. To prove that (3.4) holds true for any $\left(\mu_{1}, \mu_{2}\right) \in \mathbb{Z}_{+}^{2}$ with $\mu_{1}+\mu_{2}<m+n+1$, it suffices to prove that for any $\left(\mu_{1}, \mu_{2}\right) \in \mathbb{Z}_{+}^{2}$ such that $\mu_{1}+\mu_{2}<m+n+1$ and $\mu_{2} \geq 2 n+1$,

$$
\sum_{\left(\beta_{1}, \beta_{2}\right) \in G_{m, n}} c_{\beta_{1}, \beta_{2}}\left(\beta_{1}+\beta_{2}\right)^{\mu_{1}}\left(\beta_{1}-\beta_{2}\right)^{\mu_{2}}=0 .
$$

Note that $\left(\beta_{1}, \beta_{2}\right) \in E_{m, n}$ implies $\left|\beta_{1}-\beta_{2}\right| \leq n$. Hence, the set $E_{m, n}$ is contained in the set $\left\{\left(\beta_{1}, \beta_{2}\right) \in \mathbb{Z}^{2}: \beta_{2}=\beta_{1}-2 j, j=-n, \cdots, n\right\}$. Let $\mu_{2}$ be an integer such that $\mu_{2} \geq 2 n+1$. By using long division of polynomials, we have

$$
x_{2}^{\mu_{2}}=q_{\mu_{2}}\left(x_{1}, x_{2}\right) \prod_{j=-n}^{n}\left(x_{2}-\left(x_{1}-2 j\right)\right)+P\left(x_{1}, x_{2}\right), \quad x_{1}, x_{2} \in \mathbb{R},
$$

where $q_{\mu_{2}}$ is a polynomial in two variables and $P\left(x_{1}, x_{2}\right)$ is a linear combination of the monomials $x_{1}^{\nu_{1}} x_{2}^{\nu_{2}}$, where $\left(\nu_{1}, \nu_{2}\right) \in \mathbb{Z}_{+}^{2}, \nu_{1}+\nu_{2} \leq \mu_{2}$ and $\nu_{2}<2 n+1$. The proof of (3.6) proceeds by induction on $\mu_{2}$. It is evident that (3.6) is valid for $\mu_{2}=2 n+1$. If (3.6) is true for $\mu_{2}$, then write $P\left(x_{1}, x_{2}\right)=c\left(x_{1}\right) x_{2}^{2 n}+Q\left(x_{1}, x_{2}\right)$ such that the degree of $Q\left(x_{1}, x_{2}\right)$ in $x_{2}$ is less than $2 n$. Thus,

$$
x_{2}^{\mu_{2}+1}=x_{2} q_{\mu_{2}}\left(x_{1}, x_{2}\right) \prod_{j=-n}^{n}\left(x_{2}-\left(x_{1}-2 j\right)\right)+c\left(x_{1}\right) x_{2}^{2 n+1}+x_{2} Q\left(x_{1}, x_{2}\right) .
$$

Since (3.6) holds for $\mu_{2}=2 n+1$, it follows from the above equality that (3.6) holds for $\mu_{2}+1$. This completes the induction procedure. By setting $x_{1}=0$ and $x_{2}=0$ in (3.6), we obtain $P(0,0)=0$.

Note that for $\left|\beta_{1}\right| \leq m$ and $\left|\beta_{2}\right| \leq n,\left(\beta_{1}-\beta_{2}\right)-\left(\beta_{1}+\beta_{2}\right)-2 j=0$ for some $j$ with $-n \leq j \leq n$. It follows from (3.6) that

$$
\begin{aligned}
\sum_{\left(\beta_{1}, \beta_{2}\right) \in G_{m, n}} c_{\beta_{1}, \beta_{2}}\left(\beta_{1}+\beta_{2}\right)^{\mu_{1}} & \left(\beta_{1}-\beta_{2}\right)^{\mu_{2}} \\
& =\sum_{\left(\beta_{1}, \beta_{2}\right) \in G_{m, n}} c_{\beta_{1}, \beta_{2}}\left(\beta_{1}+\beta_{2}\right)^{\mu_{1}} P\left(\beta_{1}+\beta_{2}, \beta_{1}-\beta_{2}\right) .
\end{aligned}
$$

Note that $x_{1}^{\mu_{1}} P\left(x_{1}, x_{2}\right)$ is a linear combination of the monomials $x_{1}^{\nu_{1}} x_{2}^{\nu_{2}},\left(\nu_{1}, \nu_{2}\right) \in$ $\mathbb{Z}_{+}^{2}$ such that $\nu_{1}+\nu_{2}<m+n+1$ and $\nu_{2}<2 n+1$. Therefore, by what has been proved and $P(0,0)=0$, we have

$$
\sum_{\left(\beta_{1}, \beta_{2}\right) \in G_{m, n}} c_{\beta_{1}, \beta_{2}}\left(\beta_{1}+\beta_{2}\right)^{\mu_{1}} P\left(\beta_{1}+\beta_{2}, \beta_{1}-\beta_{2}\right)=0
$$

from which (3.5) follows. 
Let us construct the desired mask $q_{m, n}$ as follows:

$$
q_{m, n}\left(\beta_{1}, \beta_{2}\right)= \begin{cases}1, & \text { if } \beta_{1}=\beta_{2}=0 \\ c_{\beta_{1}, \beta_{2}}, & \text { if }\left(\beta_{1}, \beta_{2}\right) \in G_{m, n} \\ 0, & \text { otherwise. }\end{cases}
$$

It is evident that $q_{m, n}$ is a quincunx interpolatory mask and it follows from (3.4) that the quincunx interpolatory mask $q_{m, n}$ satisfies the sum rules of order $m+n+1$, as desired.

If there is another quincunx interpolatory mask $a$ satisfying all the conditions in Theorem 3.3, then

$$
\sum_{\left(\beta_{1}, \beta_{2}\right) \in G_{m, n}} a\left(\beta_{1}, \beta_{2}\right) p\left(\beta_{1}, \beta_{2}\right)=p(0,0) \quad \forall p \in \Pi_{m+n} .
$$

For $\left(\mu_{1}, \mu_{2}\right) \in \Gamma_{m, n}$, we have $\mu_{1}+\mu_{2}<m+n+1$; hence, it follows that

$$
\sum_{\left(\beta_{1}, \beta_{2}\right) \in G_{m, n}} a\left(\beta_{1}, \beta_{2}\right)\left(\beta_{1}+\beta_{2}\right)^{\mu_{1}}\left(\beta_{1}-\beta_{2}\right)^{\mu_{2}}=\delta\left(\mu_{1}, \mu_{2}\right) \quad \forall\left(\mu_{1}, \mu_{2}\right) \in \Gamma_{m, n} .
$$

Since the solution to the linear system of equations 3.3 is unique, we must have

$$
a\left(\beta_{1}, \beta_{2}\right)=c_{\beta_{1}, \beta_{2}}=q_{m, n}\left(\beta_{1}, \beta_{2}\right) \quad \forall\left(\beta_{1}, \beta_{2}\right) \in G_{m, n} .
$$

Hence, the quincunx interpolatory mask $a$ must be the mask $q_{m, n}$.

The above proof tells us that for a pair of nonnegative integers $m$ and $n$ such that $m+n$ is an odd integer, the quincunx interpolatory mask $q_{m, n}$ is minimally supported among all the quincunx interpolatory masks which satisfy the sum rules of order $m+n+1$. Also the uniqueness of the mask $q_{m, n}$ implies that $q_{m, n}$ is symmetric about both the axis $x_{1}=0$ and the axis $x_{2}=0$. Let $\phi_{q_{m, n}}^{T}$ be the normalized solution to the refinement equation (1.2) with the mask $q_{m, n}$ and the dilation matrix $T$ defined in (1.1). By Theorem 2.4, $\nu_{p}\left(\phi_{q_{m, n}}^{T}\right) \leq \nu_{p}\left(\phi_{b_{(m+n+1) / 2}}\right)$ for all $1 \leq p \leq \infty$, where $\phi_{b_{(m+n+1) / 2}}$ is the univariate Deslauriers-Dubuc fundamental refinable function with the mask $b_{(m+n+1) / 2}$ given by Theorem 2.1. In Section 4, all of our examples have the property that $\nu_{2}\left(\phi_{q_{m, n}}^{T}\right)=\nu_{2}\left(\phi_{b_{(m+n+1) / 2}}\right)$.

It is obvious that $q_{2 r-1,0}(j, 0)=q_{0,2 r-1}(0, j)=b_{r}(j)$ for all $j \in \mathbb{Z}$ and it is easy to verify that $\nu_{p}\left(\phi_{q_{2 r-1,0}^{T}}^{T}\right)=\nu_{p}\left(\phi_{q_{0,2 r-1}}^{T}\right)=\nu_{p}\left(\phi_{b_{r}}\right)$ for all $1 \leq p \leq \infty$ and $r \in \mathbb{N}$.

In the following we shall prove that the symbols of both $q_{2 r, 1}$ and $q_{2 r-1,2}$ are nonnegative for all $r \in \mathbb{N}$. From (1.3), it is easy to verify that $a$ is a quincunx interpolatory mask if and only if

$$
\widetilde{a}\left(e^{-i \xi_{1}}, e^{-i \xi_{2}}\right)+\widetilde{a}\left(e^{-i\left(\xi_{1}+\pi\right)}, e^{-i\left(\xi_{2}+\pi\right)}\right)=2 \quad \forall \xi_{1}, \xi_{2} \in \mathbb{R} .
$$

Theorem 3.4. For each positive integer $r$, the symbol of the quincunx interpolatory mask $q_{2 r, 1}$ defined in Theorem 3.3 satisfies

$$
\begin{aligned}
& \widetilde{q}_{2 r, 1}\left(e^{-i \xi_{1}}, e^{-i \xi_{2}}\right) \\
& \quad=\frac{(2 r) !}{2^{2 r-1} r !(r-1) !} \int_{-1}^{\cos \xi_{1}}\left(1-t^{2}\right)^{r-1}\left(1-t \cos \xi_{2}\right) d t, \quad \xi_{1}, \xi_{2} \in \mathbb{R}
\end{aligned}
$$


or equivalently, the symbol of $q_{2 r, 1}$ is

$$
\begin{aligned}
& \widetilde{q}_{2 r, 1}\left(z_{1}, z_{2}\right) \\
& \quad=\widetilde{b}_{r}\left(z_{1}\right)+(-1)^{r} \frac{(2 r) !}{2^{4 r+1} r ! r !}\left(z_{1}^{-1}-z_{1}\right)^{2 r}\left(z_{2}^{-1}+z_{2}\right), \quad z_{1}, z_{2} \in \mathbb{C} \backslash\{0\},
\end{aligned}
$$

where $b_{r}$ is the univariate interpolatory mask given in Theorem 2.1. Moreover, the symbol of $q_{2 r, 1}$ is nonnegative, i.e., $\widetilde{q_{2 r, 1}}\left(e^{-i \xi}\right) \geq 0$ for all $\xi \in \mathbb{R}^{2}$.

Proof. Let $a$ denote the mask with $\widetilde{a}\left(e^{-i \xi_{1}}, e^{-i \xi_{2}}\right)$ being the right-hand side of (3.7). To complete the proof, it suffices to prove that $a=q_{2 r, 1}$. Note that

$$
\begin{aligned}
\widetilde{a}\left(e^{-i\left(\xi_{1}+\pi\right)}, e^{-i\left(\xi_{2}+\pi\right)}\right) & =\frac{(2 r) !}{2^{2 r-1} r !(r-1) !} \int_{-1}^{-\cos \xi_{1}}\left(1-t^{2}\right)^{r-1}\left(1+t \cos \xi_{2}\right) d t \\
& =\frac{(2 r) !}{2^{2 r-1} r !(r-1) !} \int_{\cos \xi_{1}}^{1}\left(1-t^{2}\right)^{r-1}\left(1-t \cos \xi_{2}\right) d t .
\end{aligned}
$$

By induction and integration by parts, we obtain

$$
\int_{-1}^{1}\left(1-t^{2}\right)^{k} d t=\frac{2 k}{2 k+1} \int_{-1}^{1}\left(1-t^{2}\right)^{k-1} d t=\frac{2^{2 k+2} k !(k+1) !}{(2 k+2) !}, \quad k \in \mathbb{N} .
$$

Thus, we have

$$
\begin{aligned}
\widetilde{a}\left(e^{-i \xi_{1}}, e^{-i \xi_{2}}\right) & +\widetilde{a}\left(e^{-i\left(\xi_{1}+\pi\right)}, e^{-i\left(\xi_{2}+\pi\right)}\right) \\
& =\frac{(2 r) !}{2^{2 r-1} r !(r-1) !} \int_{-1}^{1}\left(1-t^{2}\right)^{r-1}\left(1-t \cos \xi_{2}\right) d t \\
& =\frac{(2 r) !}{2^{2 r-1} r !(r-1) !} \int_{-1}^{1}\left(1-t^{2}\right)^{r-1} d t=2 .
\end{aligned}
$$

Hence, $a$ is a quincunx interpolatory mask. Let $Q\left(\eta_{1}, \eta_{2}\right):=\widetilde{a}\left(e^{-i \xi_{1}}, e^{-i \xi_{2}}\right)$ with $\eta_{1}=\cos \xi_{1}$ and $\eta_{2}=\cos \xi_{2}$. Then it is easy to verify that

$$
\left.\frac{\partial^{\mu_{1}+\mu_{2}} Q\left(\eta_{1}, \eta_{2}\right)}{\partial \eta_{1}^{\mu_{1}} \partial \eta_{2}^{\mu_{2}}}\right|_{\eta_{1}=-1, \eta_{2}=-1}=0 \quad \forall\left(\mu_{1}, \mu_{2}\right) \in \mathbb{Z}_{+}^{2}, \mu_{1}+\mu_{2} \leq r .
$$

It follows that

$$
\left.\frac{\partial^{\mu_{1}+\mu_{2}} \widetilde{a}\left(e^{-i \xi_{1}}, e^{-i \xi_{2}}\right)}{\partial \xi_{1}^{\mu_{1}} \partial \xi_{2}^{\mu_{2}}}\right|_{\xi_{1}=\pi, \xi_{2}=\pi}=0 \quad \forall\left(\mu_{1}, \mu_{2}\right) \in \mathbb{Z}_{+}^{2}, \mu_{1}+\mu_{2} \leq 2 r,
$$

or equivalently, $a$ satisfies the sum rules of order $2 r+1$ with respect to the quincunx lattice. Since $a$ is symmetric about the origin and is a quincunx interpolatory mask, by the definition of sum rules, $a$ must satisfy the sum rules of order $2 r+2$. Note that $a$ is supported on $[-2 r, 2 r] \times[-1,1]$. Hence, by Theorem 3.3, $a$ must be the unique mask $q_{2 r, 1}$. By (3.7), it is evident that the symbol of $q_{2 r, 1}$ is nonnegative. By a similar argument and Theorem 2.1, we have (see Meyer [22] and Micchelli [23])

$$
\widetilde{b}_{r}\left(e^{-i \xi_{1}}\right)=\frac{(2 r) !}{2^{2 r-1} r !(r-1) !} \int_{-1}^{\cos \xi_{1}}\left(1-t^{2}\right)^{r-1} d t .
$$

Moreover,

$$
\int_{-1}^{\cos \xi_{1}}\left(1-t^{2}\right)^{r-1}\left(-t \cos \xi_{2}\right) d t=-\frac{1}{2 r}\left(1-\cos ^{2} \xi_{1}\right)^{r} \cos \xi_{2} .
$$

Therefore, $\widetilde{q}_{2 r, 1}\left(z_{1}, z_{2}\right)$ has the desired representation as given in (3.8)). 
Theorem 3.5. For each positive integer $r>1$, the symbol of the quincunx interpolatory mask $q_{2 r-1,2}$ defined in Theorem 3.3 satisfies that for any $\xi_{1}, \xi_{2} \in \mathbb{R}$,

$$
\begin{aligned}
\widetilde{q}_{2 r-1,2}\left(e^{-i \xi_{1}}, e^{-i \xi_{2}}\right)= & \frac{(2 r-1) !}{2^{2 r-1} r !(r-1) !} \int_{-1}^{\cos \xi_{1}}\left(1-t^{2}\right)^{r-2} \\
& \times\left[(2 r-2)\left(1-t \cos \xi_{2}\right)^{2}+\left(1-t^{2}\right) \sin ^{2} \xi_{2}\right] d t,
\end{aligned}
$$

or equivalently, the symbol of $q_{2 r-1,2}$ is

$$
\begin{aligned}
\widetilde{q}_{2 r-1,2} & \left(z_{1}, z_{2}\right) \\
= & \widetilde{b}_{r}\left(z_{1}\right)+\frac{(-1)^{r}(2 r) !}{2^{4 r+1} r ! r !}\left(z_{1}-z_{1}^{-1}\right)^{2 r-2} \\
& \times\left[6\left(z_{1}+z_{1}^{-1}\right)-8\left(z_{2}+z_{2}^{-1}\right)+\left(z_{1}+z_{1}^{-1}\right)\left(z_{2}^{2}+z_{2}^{-2}\right)\right],
\end{aligned}
$$

where $b_{r}$ is the mask given in Theorem 2.1. Moreover, the symbol of $q_{2 r-1,2}$ is nonnegative, i.e., $\widetilde{q_{2 r-1,2}}\left(e^{-i \xi}\right) \geq 0$ for all $\xi \in \mathbb{R}^{2}$.

Proof. Let $a$ denote the mask with $\widetilde{a}\left(e^{-i \xi_{1}}, e^{-i \xi_{2}}\right)$ being the right-hand side of (3.11). To complete the proof, it suffices to prove $a=q_{2 r-1,2}$. Note that

$$
\begin{aligned}
& \widetilde{a}\left(e^{-i\left(\xi_{1}+\pi\right)}, e^{-i\left(\xi_{2}+\pi\right)}\right) \\
& =\frac{(2 r-1) !}{2^{2 r-1} r !(r-1) !} \int_{-1}^{-\cos \xi_{1}}\left(1-t^{2}\right)^{r-2}\left[(2 r-2)\left(1+t \cos \xi_{2}\right)^{2}+\left(1-t^{2}\right) \sin ^{2} \xi_{2}\right] d t \\
& =\frac{(2 r-1) !}{2^{2 r-1} r !(r-1) !} \int_{\cos \xi_{1}}^{1}\left(1-t^{2}\right)^{r-2}\left[(2 r-2)\left(1-t \cos \xi_{2}\right)^{2}+\left(1-t^{2}\right) \sin ^{2} \xi_{2}\right] d t .
\end{aligned}
$$

Thus, from the above equality and (3.9), we obtain

$$
\begin{aligned}
& \tilde{a}\left(e^{-i \xi_{1}}, e^{-i \xi_{2}}\right)+\widetilde{a}\left(e^{-i\left(\xi_{1}+\pi\right)}, e^{-i\left(\xi_{2}+\pi\right)}\right) \\
& =\frac{(2 r-1) !}{2^{2 r-1} r !(r-1) !} \int_{-1}^{1}\left(1-t^{2}\right)^{r-2}\left[(2 r-2)\left(1-t \cos \xi_{2}\right)^{2}+\left(1-t^{2}\right) \sin ^{2} \xi_{2}\right] d t \\
& =\frac{(2 r-1) !}{2^{2 r-2} r !(r-2) !} \int_{-1}^{1}\left(1-t^{2}\right)^{r-2}\left(1+t^{2}\right) d t \\
& \quad+\frac{(2 r-1) ! \sin ^{2} \xi_{2}}{2^{2 r-1} r !(r-1) !} \int_{-1}^{1}\left(1-t^{2}\right)^{r-2}\left(1-(2 r-1) t^{2}\right) d t=2 .
\end{aligned}
$$

Therefore, it follows that $a$ is a quincunx interpolatory mask. Let $Q\left(\eta_{1}, \eta_{2}\right):=$ $\widetilde{a}\left(e^{-i \xi_{1}}, e^{-i \xi_{2}}\right)$ with $\eta_{1}=\cos \xi_{1}$ and $\eta_{2}=\cos \xi_{2}$. Then it is easy to verify that

$$
\left.\frac{\partial^{\mu_{1}+\mu_{2}} Q\left(\eta_{1}, \eta_{2}\right)}{\partial \eta_{1}^{\mu_{1}} \partial \eta_{2}^{\mu_{2}}}\right|_{\eta_{1}=-1, \eta_{2}=-1}=0 \quad \forall\left(\mu_{1}, \mu_{2}\right) \in \mathbb{Z}_{+}^{2}, \mu_{1}+\mu_{2} \leq r .
$$

Hence, it follows that

$$
\left.\frac{\partial^{\mu_{1}+\mu_{2}} \widetilde{a}\left(e^{-i \xi_{1}}, e^{-i \xi_{2}}\right)}{\partial \xi_{1}^{\mu_{1}} \partial \xi_{2}^{\mu_{2}}}\right|_{\xi_{1}=\pi, \xi_{2}=\pi}=0 \quad \forall\left(\mu_{1}, \mu_{2}\right) \in \mathbb{Z}_{+}^{2}, \mu_{1}+\mu_{2} \leq 2 r
$$

or equivalently, $a$ satisfies the sum rules of order $2 r+1$ with respect to the quincunx lattice. Since $a$ is symmetric about the origin and is a quincunx interpolatory mask, by the definition of sum rules, $a$ must satisfy the sum rules of order $2 r+2$. Note that the sequence $a$ is supported on $[1-2 r, 2 r-1] \times[-2,2]$. Therefore, by Theorem $3.3, a$ must be the unique mask $q_{2 r-1,2}$. By (3.11), it is evident that the symbol of $q_{2 r-1,2}$ is nonnegative. By (3.10) and integration by parts, $\widetilde{q}_{2 r-1,2}$ has the desired representation as given in (3.12). 
Let $M$ denote either the matrix $Q$ or $T$ defined in (1.1). Let $a$ be a quincunx interpolatory mask such that $\widetilde{a}\left(e^{-i \xi_{1}}, e^{-i \xi_{2}}\right) \geq 0$ for all $\left(\xi_{1}, \xi_{2}\right) \in \mathbb{R}^{2}$. Note that

$$
\widehat{\phi}_{a}^{M}(\xi)=\prod_{j=1}^{\infty}\left(\widetilde{a}\left(e^{-i\left(M^{T}\right)^{-j} \xi}\right) / 2\right), \quad \xi \in \mathbb{R}^{2} .
$$

For a positive integer $n$, define

$$
f_{n}(\xi):=\prod_{j=1}^{n}\left(\widetilde{a}\left(e^{-i\left(M^{T}\right)^{-j} \xi}\right) / 2\right) \chi_{[-\pi, \pi)^{2}}\left(\left(M^{T}\right)^{-n} \xi\right), \quad \xi \in \mathbb{R}^{2} .
$$

Since the symbol of $a$ is nonnegative, we have $\widehat{\phi}_{a}^{M}(\xi) \geq 0$ and $f_{n}(\xi) \geq 0$ for all $\xi \in \mathbb{R}^{2}$. Moreover, $\lim _{n \rightarrow \infty} f_{n}(\xi)=\widehat{\phi}_{a}^{M}(\xi)$ for all $\xi \in \mathbb{R}^{2}$. Since $a$ is a quincunx interpolatory mask, we have $\widetilde{a}\left(e^{-i \xi_{1}}, e^{-i \xi_{2}}\right)+\widetilde{a}\left(e^{-i\left(\xi_{1}+\pi\right)}, e^{-i\left(\xi_{2}+\pi\right)}\right)=2$. With the help of this relation, by induction on $n$ we can easily verify that $\int_{\mathbb{R}^{2}} f_{n}(\xi) d \xi=4 \pi^{2}$ for all $n \in \mathbb{N}$. Therefore, by Fatou's lemma, we have

$$
\int_{\mathbb{R}^{2}}\left|\widehat{\phi}_{a}^{M}(\xi)\right| d \xi=\int_{\mathbb{R}^{2}} \widehat{\phi}_{a}^{M}(\xi) d \xi \leq \underline{\lim }_{n \rightarrow \infty} \int_{\mathbb{R}^{2}} f_{n}(\xi) d \xi=4 \pi^{2} .
$$

So $\widehat{\phi}_{a}^{M} \in L_{1}\left(\mathbb{R}^{2}\right)$ and $\phi_{a}^{M}$ is a continuous function. If $a$ is one of the masks $q_{2 r-1,0}, q_{2 r, 1}, q_{2 r+1,2}$, then $\widetilde{a}\left(e^{-i \xi_{1}}, e^{-i \xi_{2}}\right)=0$ if and only if $\xi_{1}=(2 k+1) \pi, k \in \mathbb{Z}$. This can be proved by using (3.7) and (3.11). By (3.13), it is not difficult to demonstrate that $\sum_{\beta \in \mathbb{Z}^{2}} \widehat{\phi}_{a}^{M}(\xi+2 \pi \beta) \neq 0$ for all $\xi \in[-\pi, \pi)^{2}$. That is, the shifts of $\phi_{a}^{M}$ are stable (see [19]). Since the shifts of $\phi_{a}^{M}$ are stable and $\phi_{a}^{M}$ is continuous, by Theorem 3.4 in [14], the subdivision scheme associated with mask $a$ and the dilation matrix $M$ converges in the $L_{\infty}$ norm. Therefore, $\phi_{a}^{M}$ is a fundamental function.

\section{EXAMPLES OF QUINCUNX INTERPOLATORY MASKS}

In this section, we shall explicitly compute several examples of $q_{m, n}$. For both $p=2$ and $p=\infty$, we calculate the $L_{p}$ critical exponents of both $\phi_{q_{m, n}}^{T}$ and $\phi_{q_{m}, n}^{Q}$, where the dilation matrices $T$ and $Q$ are given in (1.1). In particular, we are interested in the following two families of quincunx interpolatory masks: for any $r \in \mathbb{N}$,

$$
\begin{gathered}
h_{r}(i, j)=q_{r, r-1}(i, j), \quad(i, j) \in \mathbb{Z}^{2}, \\
g_{r}(i, j):=\left(q_{r, r-1}(i, j)+q_{r, r-1}(j, i)\right) / 2, \quad(i, j) \in \mathbb{Z}^{2} .
\end{gathered}
$$

It is evident that both $h_{r}$ and $g_{r}$ satisfy the sum rules of optimal order $2 r$. All the masks $h_{r}$ are symmetric about both the axis $x_{1}=0$ and the axis $x_{2}=0$. All the masks $g_{r}$ are symmetric about the axis $x_{1}=0$, the axis $x_{2}=0$, and the lines $x_{1}+x_{2}=0$ and $x_{1}-x_{2}=0$. If the symbol of $h_{r}$ is nonnegative, then the symbol of $g_{r}$ is nonnegative since $2 \widetilde{g_{r}}\left(e^{-i\left(\xi_{1}, \xi_{2}\right)}\right)=\widetilde{h_{r}}\left(e^{-i\left(\xi_{1}, \xi_{2}\right)}\right)+\widetilde{h_{r}}\left(e^{-i\left(\xi_{2}, \xi_{1}\right)}\right)$ for all $\left(\xi_{1}, \xi_{2}\right) \in \mathbb{R}^{2}$. These quincunx interpolatory masks $h_{r}$ and $g_{r}$ have a close relation with the optimal interpolatory masks with the dilation matrix $M=2 I_{2}$ proposed in [15]. 
Example 4.1. The quincunx interpolatory mask $h_{2}$ is supported on $[-2,2] \times[-1,1]$ and is given by

$$
\left[\begin{array}{ccccc}
-1 / 16 & 0 & 1 / 8 & 0 & -1 / 16 \\
0 & 1 / 2 & 1 & 1 / 2 & 0 \\
-1 / 16 & 0 & 1 / 8 & 0 & -1 / 16
\end{array}\right]
$$

Then $h_{2}$ satisfies the sum rules of order 4 and by Theorem 3.4 the symbol of $h_{2}$ is nonnegative. Moreover, $\nu_{2}\left(\phi_{h_{2}}^{T}\right) \approx 2.44077, \nu_{\infty}\left(\phi_{h_{2}}^{T}\right)=2, \nu_{2}\left(\phi_{h_{2}}^{Q}\right) \approx 1.09619$, and $\nu_{\infty}\left(\phi_{h_{2}}^{Q}\right) \approx 0.47637$.

Example 4.2. The quincunx interpolatory mask $h_{3}$ is supported on $[-3,3] \times[-2,2]$ and is given by

$$
\left[\begin{array}{ccccccc}
\frac{3}{256} & 0 & -\frac{3}{256} & 0 & -\frac{3}{256} & 0 & \frac{3}{256} \\
0 & -\frac{3}{32} & 0 & \frac{3}{16} & 0 & -\frac{3}{32} & 0 \\
\frac{1}{128} & 0 & \frac{63}{128} & 1 & \frac{63}{128} & 0 & \frac{1}{128} \\
0 & -\frac{3}{32} & 0 & \frac{3}{16} & 0 & -\frac{3}{32} & 0 \\
\frac{3}{256} & 0 & -\frac{3}{256} & 0 & -\frac{3}{256} & 0 & \frac{3}{256}
\end{array}\right]
$$

Then $h_{3}$ satisfies the sum rules of order 6 , and the symbol of $h_{3}$ is nonnegative, by Theorem 3.5. Moreover, $\nu_{2}\left(\phi_{h_{3}}^{T}\right) \approx 3.17513, \nu_{\infty}\left(\phi_{h_{3}}^{T}\right) \approx 2.83008, \nu_{2}\left(\phi_{h_{3}}^{Q}\right) \approx 1.94692$, and $\nu_{\infty}\left(\phi_{h_{3}}^{Q}\right) \approx 1.28289$. Therefore, $\phi_{h_{3}}^{T}$ is a $C^{2}$ fundamental refinable function and $\phi_{h_{3}}^{Q}$ is a $C^{1}$ fundamental refinable function.

Example 4.3. The quincunx interpolatory mask $h_{4}$ is supported on $[-4,4] \times[-3,3]$ and is given by

$$
\left[\begin{array}{ccccccccc}
-\frac{5}{2048} & 0 & \frac{1}{512} & 0 & \frac{1}{1024} & 0 & \frac{1}{512} & 0 & -\frac{5}{2048} \\
0 & \frac{3}{128} & 0 & -\frac{3}{128} & 0 & -\frac{3}{128} & 0 & \frac{3}{128} & 0 \\
-\frac{3}{2048} & 0 & -\frac{57}{512} & 0 & \frac{231}{1024} & 0 & -\frac{57}{512} & 0 & -\frac{3}{2048} \\
0 & \frac{1}{64} & 0 & \frac{31}{64} & 1 & \frac{31}{64} & 0 & \frac{1}{64} & 0 \\
-\frac{3}{2048} & 0 & -\frac{57}{512} & 0 & \frac{231}{1024} & 0 & -\frac{57}{512} & 0 & -\frac{3}{2048} \\
0 & \frac{3}{128} & 0 & -\frac{3}{128} & 0 & -\frac{3}{128} & 0 & \frac{3}{128} & 0 \\
-\frac{5}{2048} & 0 & \frac{1}{512} & 0 & \frac{1}{1024} & 0 & \frac{1}{512} & 0 & -\frac{5}{2048}
\end{array}\right] .
$$

Then $h_{4}$ satisfies the sum rules of order 8 and the symbol of $h_{4}$ is nonnegative. Moreover, $\nu_{2}\left(\phi_{h_{4}}^{T}\right) \approx 3.79313, \nu_{\infty}\left(\phi_{h_{4}}^{T}\right) \approx 3.40412, \nu_{2}\left(\phi_{h_{4}}^{Q}\right) \approx 2.67072$, and $\nu_{\infty}\left(\phi_{h_{4}}^{Q}\right) \approx 2.02882$. Therefore, $\phi_{h_{4}}^{T}$ is a $C^{3}$ fundamental refinable function and $\phi_{h_{4}}^{Q}$ is a $C^{2}$ fundamental refinable function.

The symbol of each mask in Tables 1 and 2 is nonnegative. The $L_{2}$ and $L_{\infty}$ critical exponents of several quincunx fundamental refinable functions are presented in Tables 1 and 2. For the graphs and contours of several quincunx fundamental refinable functions, see Figures $1-6$. 
TABLE $1 . L_{2}$ and $L_{\infty}$ critical exponents of several quincunx fundamental refinable functions with respect to the dilation matrix $Q$.

\begin{tabular}{||c|c|c|c|c|c|c|c|c||}
\hline$r$ & $\nu_{2}\left(\phi_{h_{r}}^{Q}\right)$ & $\nu_{2}\left(\phi_{g_{r}}^{Q}\right)$ & $\nu_{2}\left(\phi_{q_{2 r-2,1}}^{Q}\right)$ & $\nu_{2}\left(\phi_{q_{2 r-3,2}}^{Q}\right)$ & $\nu_{\infty}\left(\phi_{h_{r}}^{Q}\right)$ & $\nu_{\infty}\left(\phi_{g_{r}}^{Q}\right)$ & $\nu_{\infty}\left(\phi_{q_{2 r-2,1}}^{Q}\right)$ & $\nu_{\infty}\left(\phi_{q_{2 r-3,2}}^{Q}\right)$ \\
\hline 1 & 1.09619 & 1.57764 & 1.09619 & $\mathrm{~N} / \mathrm{A}$ & 0.47637 & 0.61152 & 0.47637 & $\mathrm{~N} / \mathrm{A}$ \\
\hline 2 & 1.94692 & 2.44792 & 1.94692 & 1.94692 & 1.28289 & 1.45934 & 1.28289 & 1.28289 \\
\hline 3 & 2.67072 & 3.15425 & 1.45796 & 2.67072 & 2.02882 & 2.21896 & 1.18962 & 2.02882 \\
\hline 4 & 3.30421 & 3.76527 & 1.11848 & 1.98694 & 2.70453 & 2.90350 & 1.00883 & 1.73121 \\
\hline 5 & 3.87926 & 4.31790 & 0.89310 & 1.51068 & 3.32309 & 3.53133 & 0.84518 & 1.41349 \\
\hline 6 & 4.41608 & 4.83803 & 0.73342 & 1.19681 & 3.89836 & 4.11667 & 0.70985 & 1.15632 \\
\hline 7 & 4.92420 & 5.33983 & 0.61210 & 0.97433 & 4.44177 & 4.67061 & 0.59992 & 0.95570 \\
\hline 8 & 5.40677 & 5.83003 & 0.51707 & 0.80791 & 4.96136 & 5.20149 & 0.51043 & 0.79862 \\
\hline
\end{tabular}

TABLE 2. $L_{2}$ and $L_{\infty}$ critical exponents of several quincunx fundamental refinable functions with respect to the dilation matrix $T$.

\begin{tabular}{||c|c|c|c|c|c|c|c|c||}
\hline$r$ & $\nu_{2}\left(\phi_{h_{r}}^{T}\right)$ & $\nu_{2}\left(\phi_{g_{r}}^{T}\right)$ & $\nu_{2}\left(\phi_{q_{2 r-2,1}}^{T}\right)$ & $\nu_{2}\left(\phi_{q_{2 r-3,2}}^{T}\right)$ & $\nu_{\infty}\left(\phi_{h_{r}}^{T}\right)$ & $\nu_{\infty}\left(\phi_{g_{r}}^{T}\right)$ & $\nu_{\infty}\left(\phi_{q_{2 r-2,1}}^{T}\right)$ & $\nu_{\infty}\left(\phi_{q_{2 r-3,2}}^{T}\right)$ \\
1 & 1.5 & 1.57764 & 1.5 & $\mathrm{~N} / \mathrm{A}$ & 1.0 & 0.61152 & 1.00000 & $\mathrm{~N} / \mathrm{A}$ \\
\hline 2 & 2.44077 & 2.44792 & 2.44077 & 2.44077 & 2.0 & 1.45934 & 2.00000 & 2.00000 \\
\hline 3 & 3.17513 & 3.15425 & 3.17513 & 3.17513 & 2.83008 & 2.21896 & 2.83008 & 2.83008 \\
\hline 4 & 3.79313 & 3.76527 & 3.79313 & 3.79313 & 3.40412 & 2.90350 & 3.55113 & 3.55113 \\
\hline 5 & 4.34408 & 4.31790 & 4.34408 & 4.34408 & 3.93422 & 3.53133 & 4.19357 & 4.19357 \\
\hline 6 & 4.86202 & 4.83803 & 4.86202 & 4.86202 & 4.45232 & 4.11667 & 4.77675 & 4.77675 \\
\hline 7 & 5.36283 & 5.33983 & 5.36283 & 5.36283 & 4.95803 & 4.67061 & 5.31732 & 5.31732 \\
\hline 8 & 5.85293 & 5.83003 & 5.85293 & 5.85293 & 5.45328 & 5.20149 & 5.82944 & 5.82944 \\
\hline
\end{tabular}



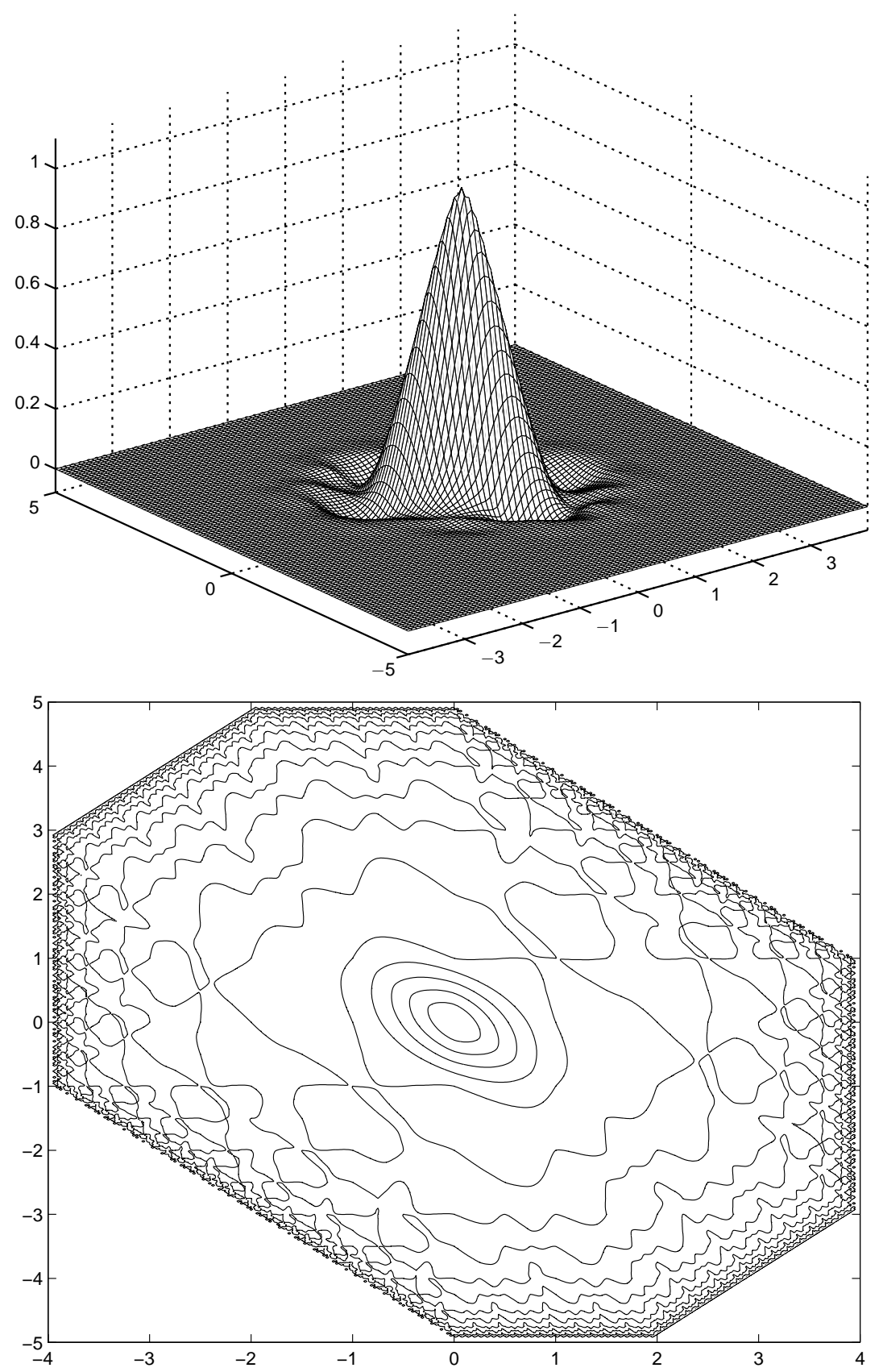

FiguRE 1. Graph and contour of the quincunx fundamental refinable function $\phi_{h_{2}}^{T}$ in Example 4.1 

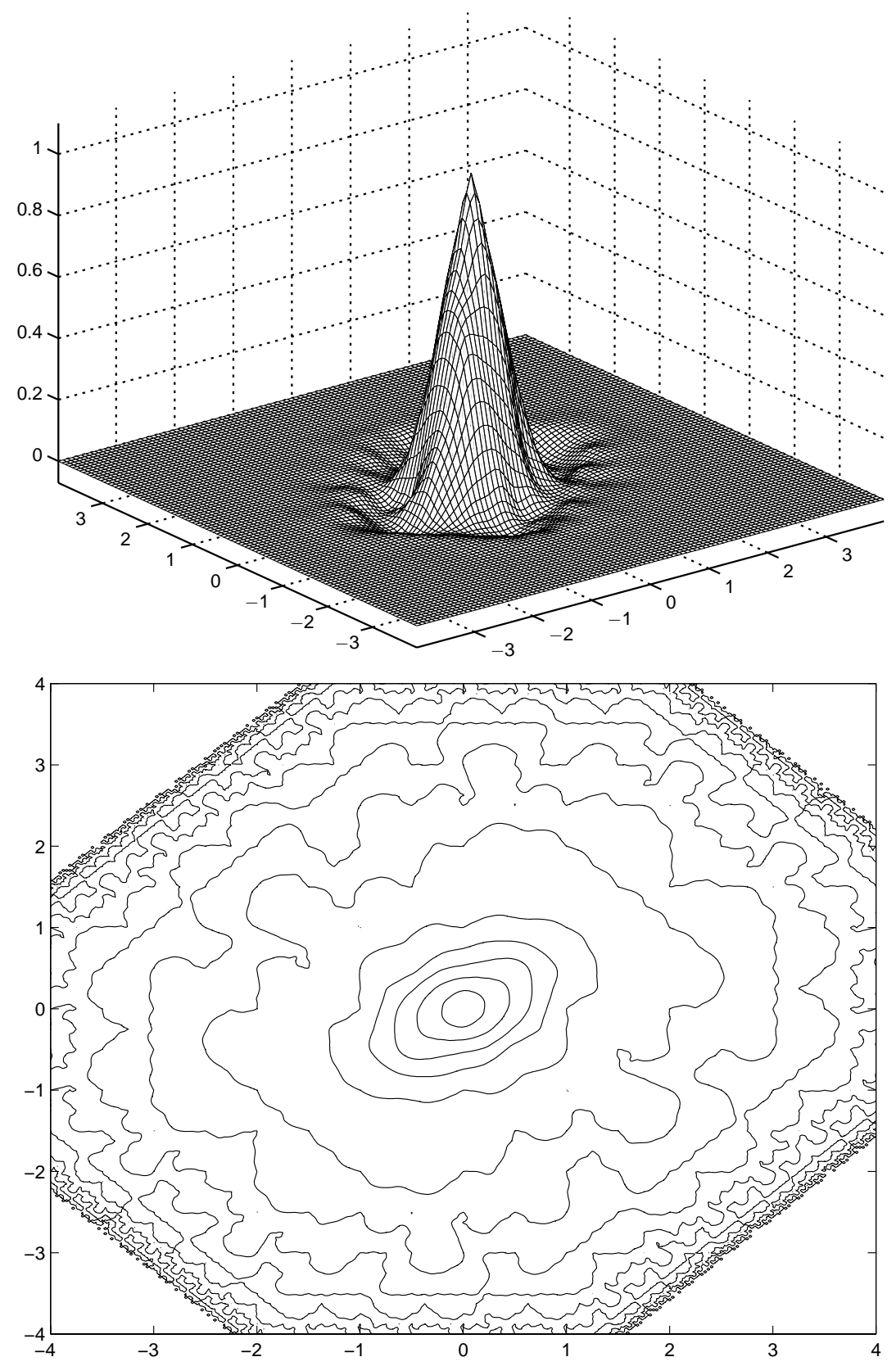

FiguRE 2. Graph and contour of the quincunx fundamental refinable function $\phi_{h_{2}}^{Q}$ in Example 4.1 

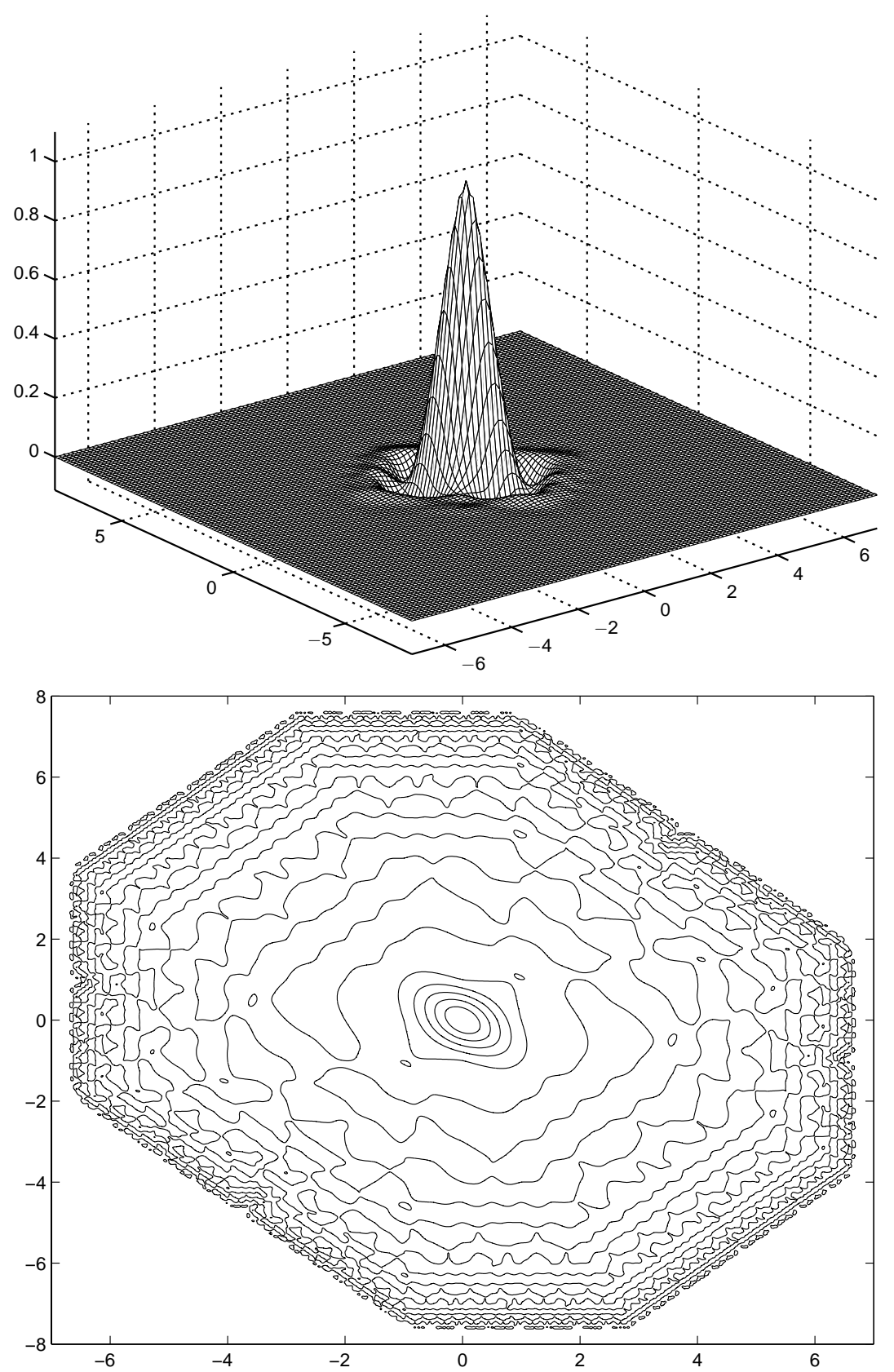

FiguRE 3. Graph and contour of the quincunx fundamental refinable function $\phi_{h_{3}}^{T}$ in Example 4.2 

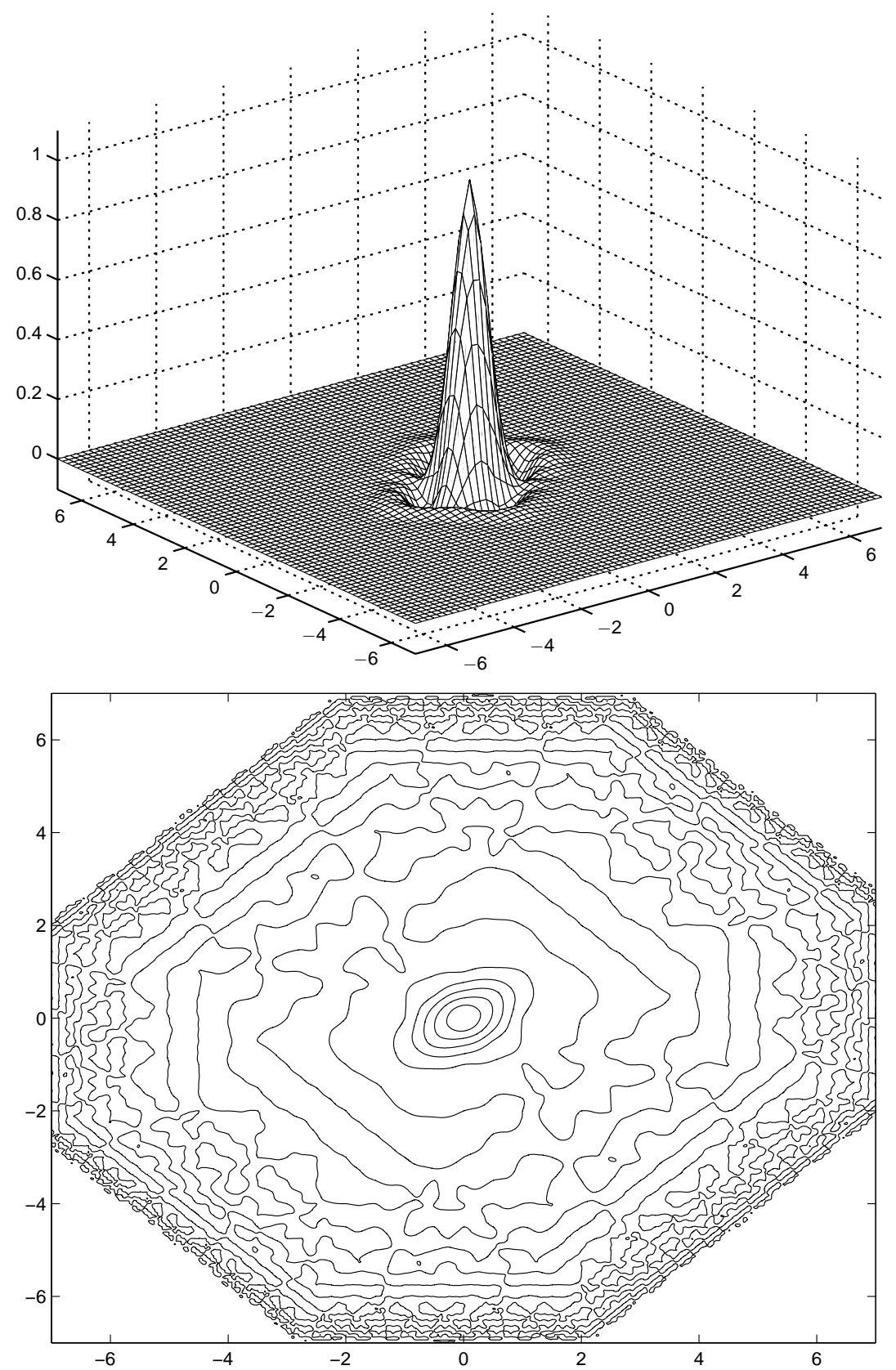

Figure 4. Graph and contour of the quincunx fundamental refinable function $\phi_{h_{3}}^{Q}$ in Example 4.2 

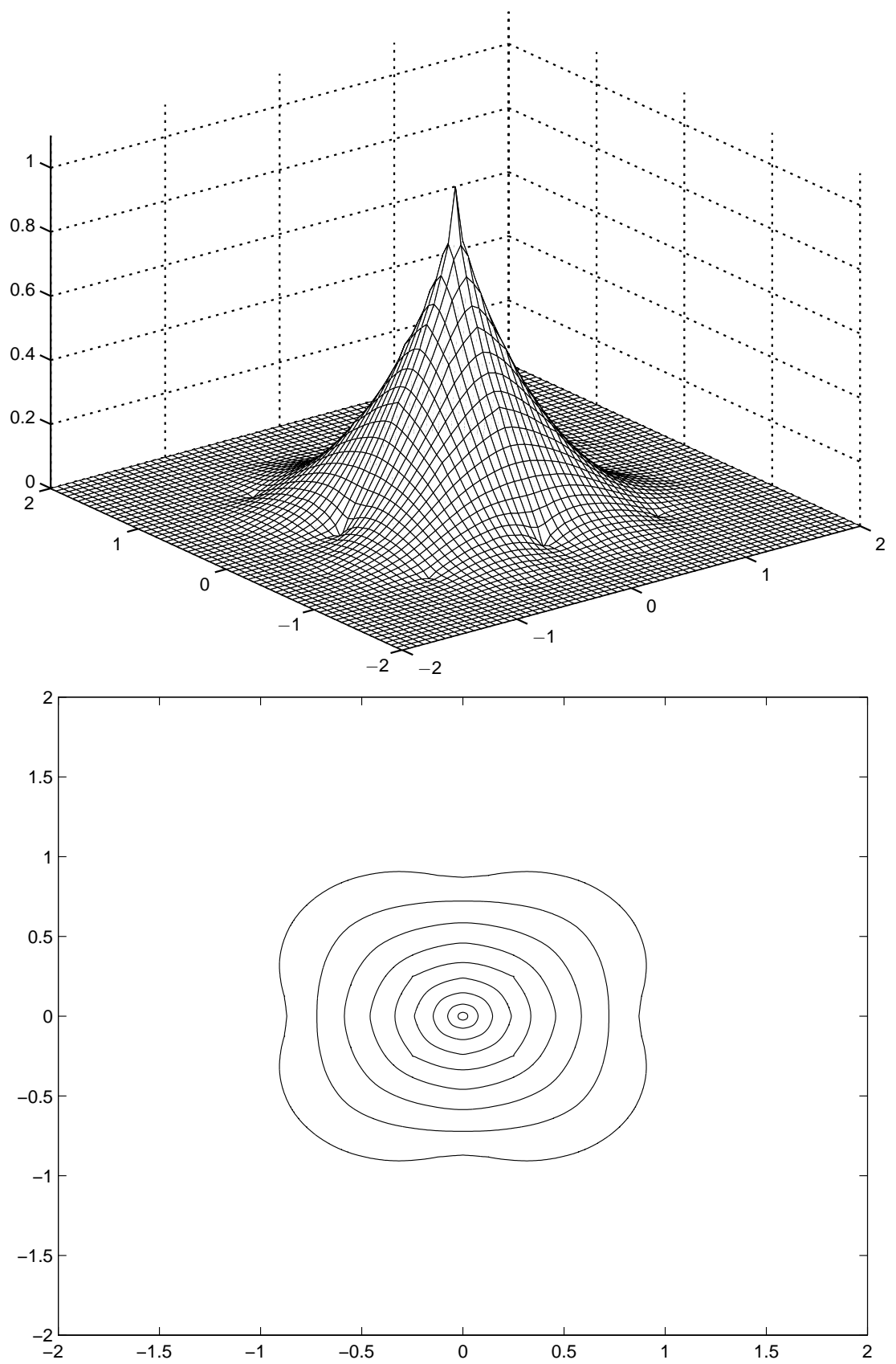

FiguRE 5. Graph and contour of the quincunx fundamental refinable function $\phi_{g_{1}}^{Q}$. 

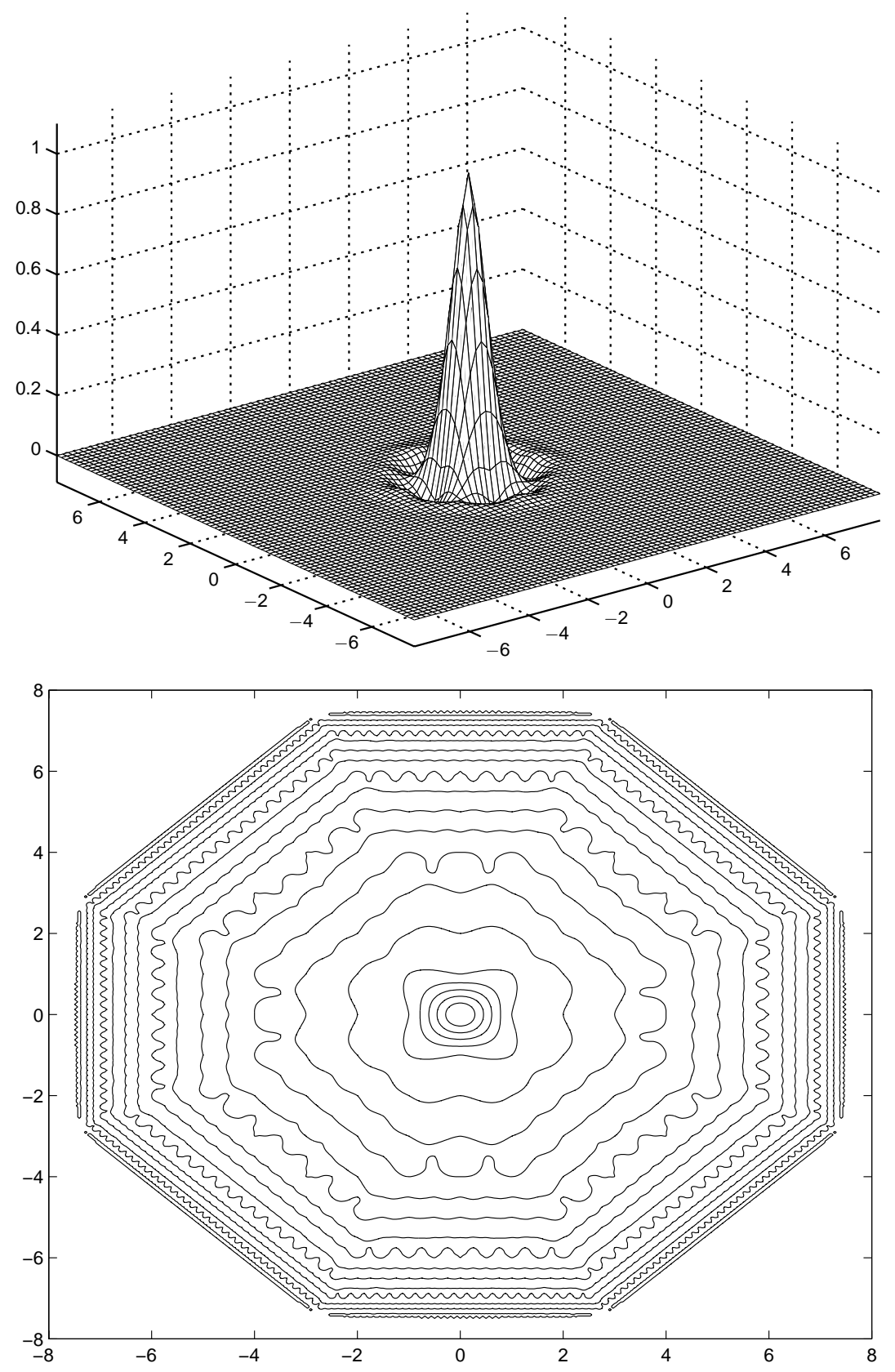

FiguRE 6. Graph and contour of the quincunx fundamental refinable function $\phi_{g_{3}}^{Q}$. 


\section{QuincunX BIORThogOnal WAVELETS}

In this section, we shall discuss how to construct quincunx biorthogonal wavelets. Throughout this section, the $2 \times 2$ matrix $M$ denotes either the dilation matrix $Q$ or the dilation matrix $T$ defined in (1.1).

A quincunx biorthogonal wavelet comes from a pair of a primal (refinable) function $\phi$ and a dual (refinable) function $\phi^{d}$ such that

$$
\phi=\sum_{\beta \in \mathbb{Z}^{2}} a(\beta) \phi(M \cdot-\beta) \text { and } \phi^{d}=\sum_{\beta \in \mathbb{Z}^{2}} a^{d}(\beta) \phi^{d}(M \cdot-\beta) \text {, }
$$

where $a$ and $a^{d}$ are finitely supported masks on $\mathbb{Z}^{2}$ and the functions $\phi$ and $\phi^{d}$ satisfy the biorthogonal condition

$$
\int_{\mathbb{R}^{2}} \overline{\phi(t+\beta)} \phi^{d}(t) d t=\delta(\beta) \quad \forall \beta \in \mathbb{Z}^{2} .
$$

From these two refinable functions, a wavelet function $\psi$ and a dual wavelet function $\psi^{d}$ are derived by

$$
\psi=\sum_{\beta \in \mathbb{Z}^{2}}(-1)^{\left|e_{2}-\beta\right|} \overline{a^{d}\left(e_{2}-\beta\right)} \phi(M \cdot-\beta)
$$

and

$$
\psi^{d}=\sum_{\beta \in \mathbb{Z}^{2}}(-1)^{\left|e_{2}-\beta\right|} \overline{a\left(e_{2}-\beta\right)} \phi^{d}(M \cdot-\beta),
$$

where $e_{2}=(0,1)^{T}$. Let $f$ be a function. For $j \in \mathbb{Z}$ and $\beta \in \mathbb{Z}^{2}$, define $f_{j, \beta}:=$ $2^{j / 2} f\left(M^{j} \cdot-\beta\right)$. Then it follows from (5.1) that

$$
\left\langle\psi_{i, \alpha}^{d}, \psi_{j, \beta}\right\rangle:=\int_{\mathbb{R}^{s}} \overline{\psi_{j, \beta}(x)} \psi_{i, \alpha}^{d}(x) d x=\delta(i-j) \delta(\alpha-\beta) \quad \forall i, j \in \mathbb{Z}, \alpha, \beta \in \mathbb{Z}^{2} .
$$

Therefore, for any $f \in L_{2}\left(\mathbb{R}^{2}\right)$, we have

$$
f=\sum_{j \in \mathbb{Z}} \sum_{\beta \in \mathbb{Z}^{2}}\left\langle f, \psi_{j, \beta}^{d}\right\rangle \psi_{j, \beta}=\sum_{j \in \mathbb{Z}} \sum_{\beta \in \mathbb{Z}^{2}}\left\langle f, \psi_{j, \beta}\right\rangle \psi_{j, \beta}^{d} .
$$

An advantage of quincunx biorthogonal wavelets rests on the fact that the associated wavelet function $\psi$ and the dual wavelet function $\psi^{d}$ can be easily obtained. If the dilation matrix is $2 I_{2}$, then there are three associated wavelet functions and three dual wavelet functions; hence, there is no easy way of deriving wavelets from the primal and dual refinable functions.

A necessary condition for the functions $\phi$ and $\phi^{d}$ to satisfy the biorthogonal condition (5.1) is that their masks $a$ and $a^{d}$ satisfy the discrete biorthogonal relation

$$
\sum_{\beta \in \mathbb{Z}^{2}} \overline{a(\beta+M \alpha)} a^{d}(\beta)=|\operatorname{det} M| \delta(\alpha) \quad \forall \alpha \in \mathbb{Z}^{2} .
$$

Let $a$ be a finitely supported sequence on $\mathbb{Z}^{2}$ such that $\sum_{\beta \in \mathbb{Z}^{2}} a(\beta)=|\operatorname{det} M|$. If there exists a finitely supported sequence $a^{d}$ on $\mathbb{Z}^{2}$ such that (5.2) holds true, then the mask $a$ is called a primal mask and $a^{d}$ is called a dual mask of $a$. Note that $Q \mathbb{Z}^{2}=T \mathbb{Z}^{2}$ where the matrices $Q$ and $T$ are given in (1.1). If $a$ and $a^{d}$ satisfy (5.2) with the dilation matrix $Q$, then (5.2) also holds true with the dilation matrix $T$, and vice versa. Therefore, in this section, we shall deal with the dilation matrix $Q$ only. 
Let $a$ and $a^{d}$ be a pair of primal and dual masks. Let $\phi_{a}^{M}$ and $\phi_{a^{d}}^{M}$ be the normalized solutions to the refinement equations with the dilation matrix $M$ and the masks $a$ and $a^{d}$, respectively. Then the functions $\phi_{a}^{M}$ and $\phi_{a^{d}}^{M}$ lie in $L_{2}\left(\mathbb{R}^{2}\right)$ and satisfy the biorthogonal condition (5.1) if and only if the subdivision schemes associated with the dilation matrix $M$ and the masks $a$ and $a^{d}$ converge in the $L_{2}$ norm. See [14] for a characterization of $L_{p}(1 \leq p \leq \infty)$ convergence of subdivision schemes with a general dilation matrix. The concept of vanishing moments of a quincunx biorthogonal wavelet plays an important role in applications. See [2, 4, 6. 17. and references therein for discussions on vanishing moments and their relation to sum rules. Given a primal mask, it is desirable to construct a dual mask with high order of sum rules and relatively small support. Given an interpolatory mask as a primal mask with the dilation matrix $M=2 I_{2}$, a coset by coset (CBC) algorithm was proposed in 13 to give dual masks with arbitrary order of sum rules. The CBC algorithm was later generalized to general primal masks in 2].

Given $\mu=\left(\mu_{1}, \mu_{2}\right) \in \mathbb{Z}_{+}^{2}$, its factorial is $\mu$ ! := $\mu_{1}$ ! $\mu_{2}$ !. For $\nu=\left(\nu_{1}, \nu_{2}\right) \in \mathbb{Z}_{+}^{2}$, by $\nu \leq \mu$ we mean $\nu_{1} \leq \mu_{1}$ and $\nu_{2} \leq \mu_{2}$. By $\nu<\mu$ we mean $\nu \leq \mu$ and $\nu \neq \mu$.

We shall employ the CBC algorithm to construct quincunx biorthogonal wavelets. The reader is referred to [2, 13] for more details about the CBC algorithm.

Theorem 5.1. Let $Q$ be the dilation matrix defined in (1.1) and let a be a primal mask satisfying $\sum_{\beta \in \mathbb{Z}^{2}} a(Q \beta)=\sum_{\beta \in \mathbb{Z}^{2}} a\left(e_{2}+Q \beta\right)=1$ where $e_{2}=(0,1)^{T}$. Let $a^{d}$ be a dual mask of $a$. Define

$$
h^{a}(\mu):=2^{-1} \sum_{\beta \in \mathbb{Z}^{2}} a^{d}(\beta)\left(Q^{-1} \beta\right)^{\mu}, \quad \mu \in \mathbb{Z}_{+}^{2} .
$$

If $a^{d}$ satisfies the sum rules of order $k$ for some positive integer $k$, then $h^{a}(0)=1$ and

$$
\begin{aligned}
h^{a}(\mu)=\delta(\mu)-\frac{1}{2} \sum_{0 \leq \nu<\mu}(-1)^{|\mu-\nu|} & \frac{\mu !}{\nu !(\mu-\nu) !} h^{a}(\nu) \\
& \times \sum_{\beta \in \mathbb{Z}^{2}} \overline{a(\beta)}\left(Q^{-1} \beta\right)^{\mu-\nu}, \quad|\mu|<k .
\end{aligned}
$$

Conversely, if $h^{a}(0)=1$ and $h^{a}(\mu)(|\mu|<k)$ are given by (15.3), and if

$$
\sum_{\beta \in \mathbb{Z}^{2}} a^{d}\left(e_{2}+Q \beta\right)\left(Q^{-1} e_{2}+\beta\right)^{\nu}=h^{a}(\nu) \quad \forall|\nu|<k,
$$

then $a^{d}$ satisfies the sum rules of order $k$.

Proof. Let $\Omega:=\left\{0, e_{2}\right\}$. Since $a^{d}$ is a dual mask of $a$, it follows from (5.2) that

$$
\begin{aligned}
2 \delta(\mu) & =\sum_{\alpha \in \mathbb{Z}^{2}} \sum_{\beta \in \mathbb{Z}^{2}} \overline{a(\beta-Q \alpha)} a^{d}(\beta) \alpha^{\mu} \\
& =\sum_{\varepsilon \in \Omega} \sum_{\alpha \in \mathbb{Z}^{2}} \sum_{\beta \in \mathbb{Z}^{2}} \overline{a(\varepsilon+Q \beta-Q \alpha)} a^{d}(\varepsilon+Q \beta) \alpha^{\mu}, \quad \mu \in \mathbb{Z}_{+}^{2} .
\end{aligned}
$$

Note that $(x-y)^{\mu}=\sum_{0 \leq \nu \leq \mu}(-1)^{|\mu-\nu|}\left(\begin{array}{l}\mu \\ \nu\end{array}\right) x^{\nu} y^{\mu-\nu}$, where $\left(\begin{array}{l}\mu \\ \nu\end{array}\right):=\frac{\mu !}{\nu !(\mu-\nu) !}$. Consequently,

$$
\alpha^{\mu}=\sum_{0 \leq \nu \leq \mu}(-1)^{|\mu-\nu|}\left(\begin{array}{l}
\mu \\
\nu
\end{array}\right)\left(Q^{-1} \varepsilon+\beta-\alpha\right)^{\mu-\nu}\left(Q^{-1} \varepsilon+\beta\right)^{\nu}
$$


Hence, we obtain

$$
\begin{aligned}
2 \delta(\mu)=\sum_{0 \leq \nu \leq \mu}(-1)^{|\mu-\nu|}\left(\begin{array}{l}
\mu \\
\nu
\end{array}\right) & \sum_{\varepsilon \in \Omega} \sum_{\alpha \in \mathbb{Z}^{2}} \overline{a(\varepsilon+Q \alpha)}\left(Q^{-1} \varepsilon+\alpha\right)^{\mu-\nu} \\
& \times \sum_{\beta \in \mathbb{Z}^{2}} a^{d}(\varepsilon+Q \beta)\left(Q^{-1} \varepsilon+\beta\right)^{\nu} .
\end{aligned}
$$

Suppose $a^{d}$ satisfies the sum rules of order $k$. Then by (2.2) we have

$$
\sum_{\beta \in \mathbb{Z}^{2}} a^{d}(Q \beta) \beta^{\nu}=\sum_{\beta \in \mathbb{Z}^{2}} a^{d}\left(e_{2}+Q \beta\right)\left(Q^{-1} e_{2}+\beta\right)^{\nu}=h^{a}(\nu) \quad \forall|\nu|<k .
$$

This together with (5.5) yields

$$
\begin{aligned}
2 \delta(\mu)= & \sum_{0 \leq \nu \leq \mu}(-1)^{|\mu-\nu|}\left(\begin{array}{l}
\mu \\
\nu
\end{array}\right) h^{a}(\nu) \\
& \times \sum_{\varepsilon \in \Omega} \sum_{\alpha \in \mathbb{Z}^{2}} \overline{a(\varepsilon+Q \alpha)}\left(Q^{-1} \varepsilon+\alpha\right)^{\mu-\nu}, \quad|\mu|<k,
\end{aligned}
$$

from which (5.3) follows at once.

Now suppose $h^{a}(0)=1$ and $h^{a}(\mu)(|\mu|<k)$ are given by the recursive relation (5.3). Subtracting (5.6) from (5.5), we obtain

$$
\begin{aligned}
\sum_{0 \leq \nu \leq \mu}(-1)^{|\mu-\nu|}\left(\begin{array}{l}
\mu \\
\nu
\end{array}\right) \sum_{\varepsilon \in \Omega} \sum_{\alpha \in \mathbb{Z}^{2}} & \overline{a(\varepsilon+Q \alpha)}\left(Q^{-1} \varepsilon+\alpha\right)^{\mu-\nu} \\
\times & {\left[\sum_{\beta \in \mathbb{Z}^{2}} a^{d}(\varepsilon+Q \beta)\left(Q^{-1} \varepsilon+\beta\right)^{\nu}-h^{a}(\nu)\right]=0 . }
\end{aligned}
$$

If (5.4) holds, then it follows from the above equality that

$$
\sum_{0 \leq \nu \leq \mu}(-1)^{|\mu-\nu|}\left(\begin{array}{l}
\mu \\
\nu
\end{array}\right) \sum_{\alpha \in \mathbb{Z}^{2}} \overline{a(Q \alpha)} \alpha^{\mu-\nu}\left[\sum_{\beta \in \mathbb{Z}^{2}} a^{d}(Q \beta) \beta^{\nu}-h^{a}(\nu)\right]=0 \quad \forall|\mu|<k .
$$

Note that $\sum_{\alpha \in \mathbb{Z}^{2}} \overline{a(Q \alpha)}=1$, by induction on $\mu$ we deduce that

$$
\sum_{\beta \in \mathbb{Z}^{2}} a^{d}(Q \beta) \beta^{\nu}-h^{a}(\nu)=0 \quad \forall|\nu|<k .
$$

Therefore, $a^{d}$ satisfies the sum rules of order $k$. The proof is complete.

Suppose $\sum_{\beta \in \mathbb{Z}^{2}} a(Q \beta)=\sum_{\beta \in \mathbb{Z}^{2}} a\left(e_{2}+Q \beta\right)=1$. Let $b$ be a dual mask of $a$. Construct a new sequence $a^{d}$ as follows:

$$
a^{d}(Q \alpha):=b(Q \alpha)-\sum_{\beta \in \mathbb{Z}^{2}} \overline{a\left(e_{2}+Q \beta-Q \alpha\right)} c_{\beta}, \quad \alpha \in \mathbb{Z}^{2},
$$

and

$$
a^{d}\left(e_{2}+Q \alpha\right):=b\left(e_{2}+Q \alpha\right)+\sum_{\beta \in \mathbb{Z}^{2}} \overline{a(Q \beta-Q \alpha)} c_{\beta}, \quad \alpha \in \mathbb{Z}^{2},
$$

where $c_{\beta}\left(\beta \in \mathbb{Z}^{2}\right)$ are parameters such that $c_{\beta}=0$ except for finitely many $\beta$. Then it is easy to verify that $a^{d}$ is also a dual mask of $a$. This procedure is the 
so-called lifting scheme (see [26]). In order for $a^{d}$ to satisfy the sum rules of order $k$, we choose the parameters $c_{\beta}\left(\beta \in \mathbb{Z}^{2}\right)$ in such a way that (5.4) is satisfied. Let

$$
g(\mu):=\sum_{\beta \in \mathbb{Z}^{2}} c_{\beta}\left(Q^{-1} e_{2}+\beta\right)^{\mu}, \quad|\mu|<k .
$$

Then by (5.4) and (5.8) we have

$$
\begin{aligned}
h^{a}(\mu) & =\sum_{\alpha \in \mathbb{Z}^{2}} a^{d}\left(e_{2}+Q \alpha\right)\left(Q^{-1} e_{2}+\alpha\right)^{\mu} \\
& =\sum_{\alpha \in \mathbb{Z}^{2}} b\left(e_{2}+Q \alpha\right)\left(Q^{-1} e_{2}+\alpha\right)^{\mu}+\sum_{\alpha \in \mathbb{Z}^{2}} \sum_{\beta \in \mathbb{Z}^{2}} \overline{a(Q \beta-Q \alpha)} c_{\beta}\left(Q^{-1} e_{2}+\alpha\right)^{\mu} .
\end{aligned}
$$

Moreover,

$$
\begin{aligned}
\sum_{\alpha \in \mathbb{Z}^{2}} & \sum_{\beta \in \mathbb{Z}^{2}} \overline{a(Q \beta-Q \alpha)} c_{\beta}\left(Q^{-1} e_{2}+\alpha\right)^{\mu} \\
& =\sum_{\alpha \in \mathbb{Z}^{2}} \sum_{\beta \in \mathbb{Z}^{2}} \overline{a(Q \beta-Q \alpha)} c_{\beta}\left(Q^{-1} e_{2}+\beta-(\beta-\alpha)\right)^{\mu} \\
& =\sum_{0 \leq \nu \leq \mu}(-1)^{|\mu-\nu|} \frac{\mu !}{\nu !(\mu-\nu) !} \sum_{\beta \in \mathbb{Z}^{2}} \sum_{\alpha \in \mathbb{Z}^{2}} \overline{a(Q \beta-Q \alpha)}(\beta-\alpha)^{\mu-\nu} c_{\beta}\left(Q^{-1} e_{2}+\beta\right)^{\nu} \\
& =\sum_{0 \leq \nu \leq \mu}(-1)^{|\mu-\nu|} \frac{\mu !}{\nu !(\mu-\nu) !} \sum_{\alpha \in \mathbb{Z}^{2}} \overline{a(Q \alpha)} \alpha^{\mu-\nu} \sum_{\beta \in \mathbb{Z}^{2}} c_{\beta}\left(Q^{-1} e_{2}+\beta\right)^{\nu} \\
& =\sum_{0 \leq \nu \leq \mu}(-1)^{|\mu-\nu|} \frac{\mu !}{\nu !(\mu-\nu) !} g(\nu) \sum_{\alpha \in \mathbb{Z}^{2}} \overline{a(Q \alpha)} \alpha^{\mu-\nu} .
\end{aligned}
$$

Therefore, $g(\mu)(|\mu|<k)$ are determined by the recursive relation

$$
\begin{aligned}
g(\mu)=h^{a}(\mu)- & \sum_{\beta \in \mathbb{Z}^{2}} b\left(e_{2}+Q \beta\right)\left(Q^{-1} e_{2}+\beta\right)^{\mu} \\
& -\sum_{0 \leq \nu<\mu}(-1)^{|\mu-\nu|} \frac{\mu !}{\nu !(\mu-\nu) !} g(\nu) \sum_{\beta \in \mathbb{Z}^{2}} \overline{a(Q \beta)} \beta^{\mu-\nu} .
\end{aligned}
$$

Let $g(\mu)(|\mu|<k)$ be given in (5.10). Choose parameters $c_{\beta}\left(\beta \in \mathbb{Z}^{2}\right)$ such that (5.9) holds. Let $a^{d}$ be given by (5.7) and (5.8). Then from the above discussion it is easy to see that $a^{d}$ satisfies (5.4). By Theorem 5.1, $a^{d}$ satisfies the sum rules of order $k$. Therefore, to obtain a dual mask $a^{d}$ of $a$ satisfying the sum rules of order $k$, we only need to choose the parameters $c_{\beta}\left(\beta \in \mathbb{Z}^{2}\right)$ in such a way that (5.9) is satisfied.

Let $a$ be a primal mask which is symmetric about the origin. Given a dual mask $b$ of $a$, then without loss of generality we may assume that $b$ is also symmetric about the origin.

Let $c_{\beta}=d_{\gamma}\left(\gamma=e_{2}+Q \beta, \gamma \in G_{k, k-1}\right)$, where the set $G_{k, k-1}$ is defined in (3.2). The following result gives a particular choice of the parameters $d_{\gamma}\left(\gamma \in G_{k, k-1}\right)$ such that the dual mask $a^{d}$ satisfies the sum rules of order $2 k$.

Theorem 5.2 (A CBC algorithm for primal masks symmetric about the origin). Let $Q$ be the dilation matrix in (1.1). Let $e_{2}=(0,1)^{T}$. Let a be a finitely supported primal mask on $\mathbb{Z}^{2}$ such that $\sum_{\beta \in \mathbb{Z}^{2}} a(Q \beta)=\sum_{\beta \in \mathbb{Z}^{2}} a\left(e_{2}+Q \beta\right)=1$ and $a(-\beta)=a(\beta)$ for all $\beta \in \mathbb{Z}^{2}$. Let $b$ be a dual mask of a with the dilation matrix $Q$ such that $b$ is symmetric about the origin. 
1. Let $k$ be a fixed positive integer. Compute the values $h^{a}(\mu)$ for $\mu \in \mathbb{Z}_{+}^{2}$ and $|\mu|<2 k$ by the recursive formula (5.3);

2. Compute the values $g(\mu)$ for $\mu \in \mathbb{Z}_{+}^{2}$ and $|\mu|<2 k$ by the recursive formula (5.10);

3. Then there is a unique solution $\left\{d_{\gamma}: \gamma \in G_{k, k-1}\right\}$ to the linear system of equations

$$
\sum_{\gamma \in G_{k, k-1}} d_{\gamma}\left(Q^{-1} \gamma\right)^{\mu}=g(\mu) \quad \forall \mu \in \Gamma_{k}^{0}
$$

where the set $\Gamma_{k}^{0}$ is defined in (3.1) and the set $G_{k, k-1}$ is defined in (3.2);

4. Construct a mask a coset by coset as follows:

$$
a^{d}(Q \alpha):=b(Q \alpha)-\sum_{\gamma \in G_{k, k-1}} \overline{a(\gamma-Q \alpha)} d_{\gamma}, \quad \alpha \in \mathbb{Z}^{2},
$$

and

$$
a^{d}\left(e_{2}+Q \alpha\right):=b\left(e_{2}+Q \alpha\right)+\sum_{\gamma \in G_{k, k-1}} \overline{a\left(\gamma-e_{2}-Q \alpha\right)} d_{\gamma}, \quad \alpha \in \mathbb{Z}^{2} .
$$

Then the mask $a^{d}$ is a dual mask of the given mask a with the dilation matrix $Q$ (or the dilation matrix $T), a^{d}$ satisfies the sum rules of order $2 k$ and $a^{d}$ is symmetric about the origin.

Proof. From the definition of $a^{d}$ in Step 4, it is easy to verify that $a^{d}$ is a dual mask of $a$ with the dilation matrix $Q$. To prove that $a^{d}$ satisfies the sum rules of order $2 k$, from the preceding discussion, it suffices to verify

$$
\sum_{\gamma \in G_{k, k-1}} d_{\gamma}\left(Q^{-1} \gamma\right)^{\mu}=g(\mu) \quad \forall \mu \in \mathbb{Z}_{+}^{2},|\mu|<2 k .
$$

Note that the existence and uniqueness of a solution to the linear system of equations in Step 3 are guaranteed by Lemma 3.1. Since $a(\beta)=a(-\beta)$ and $b(\beta)=b(-\beta)$ for all $\beta \in \mathbb{Z}^{2}$, it is easy to verify that $h^{a}(\mu)=0$ and $g(\mu)=0$ for all $\mu \in \mathbb{Z}_{+}^{2}$ such that $|\mu|$ is an odd integer.

Note that the set $G_{k, k-1}$ is symmetric about the origin and $g(\mu)=0$ for all $\mu \in \mathbb{Z}_{+}^{2}$ such that $|\mu|$ is an odd integer. The uniqueness of the solution to the linear system of equations in Step 3 implies that $d_{-\gamma}=d_{\gamma}$ for all $\gamma \in G_{k, k-1}$. Therefore,

$$
\begin{aligned}
\sum_{\gamma \in G_{k, k-1}} d_{\gamma}\left(Q^{-1} \gamma\right)^{\mu} & =\sum_{\gamma \in G_{k, k-1}} d_{-\gamma}(-1)^{|\mu|}\left(Q^{-1} \gamma\right)^{\mu} \\
& =(-1)^{|\mu|} \sum_{\gamma \in G_{k, k-1}} d_{\gamma}\left(Q^{-1} \gamma\right)^{\mu}, \quad \mu \in \mathbb{Z}_{+}^{2}
\end{aligned}
$$

It follows that

$$
\sum_{\gamma \in G_{k, k-1}} d_{\gamma}\left(Q^{-1} \gamma\right)^{\mu}=0=g(\mu) \quad \forall \mu=(0,2 j-1), j=1, \cdots, k .
$$

Hence, (5.11) follows at once from the above equality and the equality in Step 3. By the uniqueness of the solution in Step $3, a^{d}$ is symmetric about the origin. 

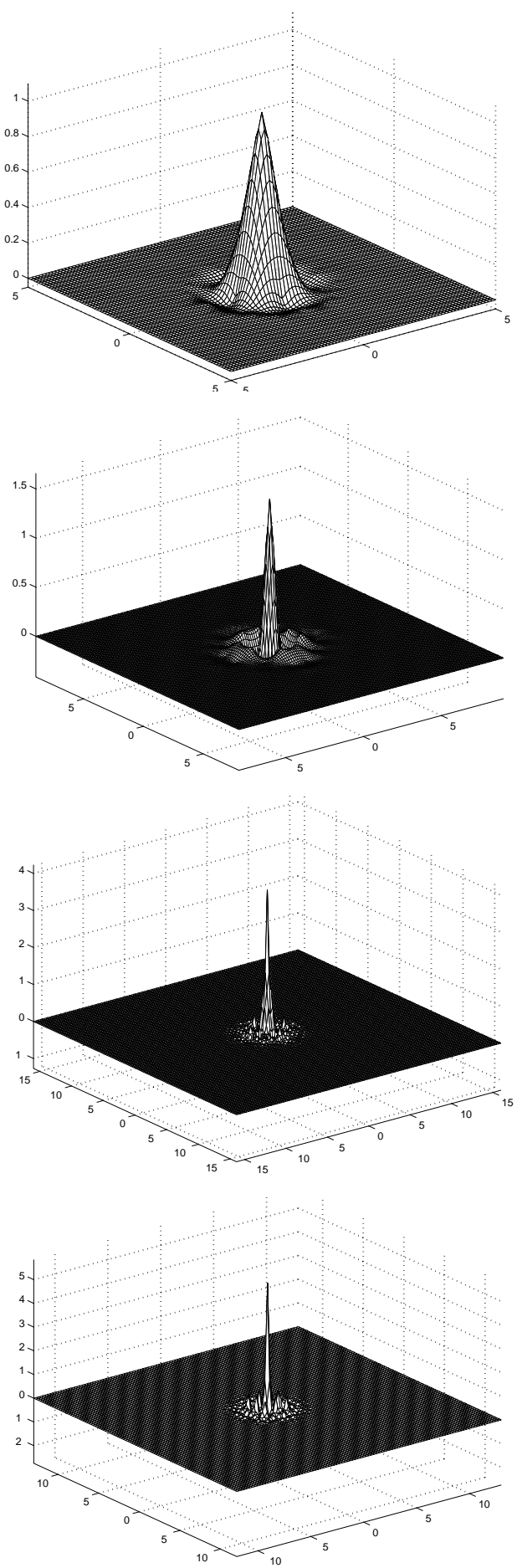

Figure 7 . The scaling function $\phi_{g_{2}}^{Q}$, the wavelet function $\psi$, the dual scaling function $\phi_{\left(g_{2}\right)_{4}^{s}}^{Q}$, and the dual wavelet function $\psi^{d}$. 
Remark. If $a$ is a quincunx interpolatory mask, then we always take $b=2 \delta$ by default in Theorem 5.2 If a primal mask $a$ is symmetric about the origin, by $(a)_{k}^{o}$ we denote the dual mask constructed by the CBC algorithm in Theorem 5.2 such that $(a)_{k}^{o}$ satisfies the sum rules of order $2 k$. When a primal mask $a$ is symmetric about the axes $x_{1}=0$ and $x_{2}=0$ we may assume that the initial mask $b$ in Theorem 5.2 is also symmetric about the axes $x_{1}=0$ and $x_{2}=0$. By the uniqueness and the symmetry of the set $G_{k, k-1}$ in Step 3, the resulting dual mask $a^{d}$ must be also symmetric about the axes $x_{1}=0$ and $x_{2}=0$. Furthermore, when a primal mask $a$ is symmetric about the axes $x_{1}=0, x_{2}=0$ and the lines $x_{1}=x_{2}$ and $x_{1}=-x_{2}$, we may assume that the initial mask $b$ in Theorem 5.2 possesses the same symmetry. We modify the dual mask $a^{d}$ in Theorem 5.2 as follows:

$$
(a)_{k}^{s}\left(\beta_{1}, \beta_{2}\right):=\left[a^{d}\left(\beta_{1}, \beta_{2}\right)+a^{d}\left(\beta_{2}, \beta_{1}\right)\right] / 2, \quad\left(\beta_{1}, \beta_{2}\right) \in \mathbb{Z}^{2} .
$$

Then the resulting mask $(a)_{k}^{s}$ is a dual mask of $a$ such that $(a)_{k}^{s}$ satisfies the sum rules of order $2 k$ and $(a)_{k}^{s}$ is symmetric about the axes $x_{1}=0, x_{2}=0$, and the lines $x_{1}=x_{2}$ and $x_{1}=-x_{2}$. In passing, we mention that the set $G_{k, k-1}$ in Theorem 5.2 can be replaced by $G_{k-1, k}$.

Let us apply Theorem 5.2 to the quincunx interpolatory masks $h_{r}$ and $g_{r}$. From Theorem [5.2, we see that each $\left(h_{r}\right)_{k}^{o}$ is supported on $[2-k-r, r+k-2] \times$ $[-k-r, r+k]$ and satisfies the sum rules of order $2 k$. Similarly, each $\left(g_{r}\right)_{k}^{s}$ is supported on $[-k-r, r+k]^{2}$ and satisfies the sum rules of order $2 k$. By computation we find that the symbols of all the dual masks in Tables 3 and 4 are nonnegative.
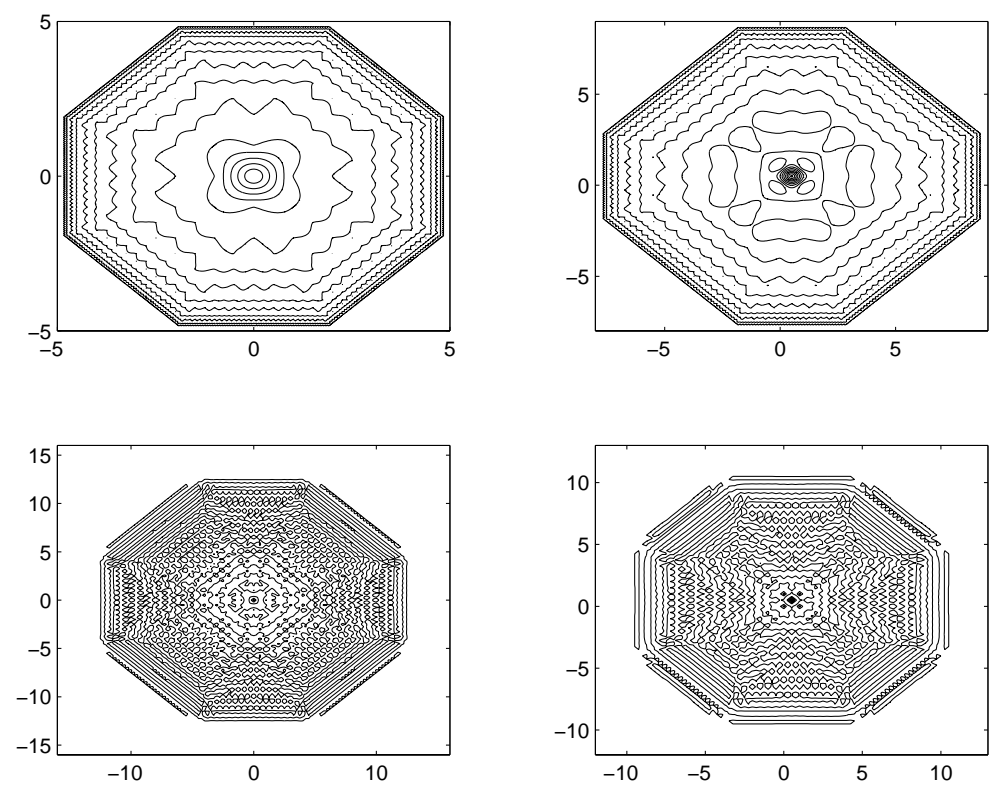

Figure 8. The contours of the scaling function $\phi_{g_{2}}^{Q}$, the wavelet function $\psi$, the dual scaling function $\phi_{\left(g_{2}\right)_{4}^{s}}^{Q}$, and the dual wavelet function $\psi^{d}$. 
TABLE 3. The $L_{\infty}$ critical exponents of several dual refinable functions $\phi_{\left(h_{r}\right)_{k}^{o}}^{T}$ and $\phi_{\left(h_{r}\right)_{k}^{o}}^{Q}$ for $r=1, \cdots, 4$ and $k=2, \cdots, 8$ which are constructed by the CBC algorithm in Theorem 5.2 ,

\begin{tabular}{||c|c|c|c|c|c|c|c|c||}
\hline$k$ & $\nu_{\infty}\left(\phi_{\left(h_{1}\right)_{k}^{o}}^{Q}\right)$ & $\nu_{\infty}\left(\phi_{\left(h_{2}\right)_{k}^{o}}^{Q}\right)$ & $\nu_{\infty}\left(\phi_{\left(h_{3}\right)_{k}^{o}}^{Q}\right)$ & $\nu_{\infty}\left(\phi_{\left(h_{4}\right)_{k}^{o}}^{Q}\right)$ & $\nu_{\infty}\left(\phi_{\left(h_{1}\right)_{k}^{o}}^{T}\right)$ & $\nu_{\infty}\left(\phi_{\left(h_{2}\right)_{k}^{o}}^{T}\right)$ & $\nu_{\infty}\left(\phi_{\left(h_{3}\right)_{k}^{o}}^{T}\right)$ & $\nu_{\infty}\left(\phi_{\left(h_{4}\right)_{k}^{o}}^{T}\right)$ \\
2 & -0.53805 & -0.54301 & -0.45331 & -0.41055 & 0.10754 & 0.19197 & 0.28003 \\
0.32808 \\
\hline 3 & -0.00096 & 0.04915 & 0.021589 & 0.138024 & 0.36007 & 0.58894 & 0.61981 & 0.74430 \\
\hline 4 & 0.522967 & 0.54310 & 0.587553 & 0.563213 & 0.68497 & 0.99869 & 1.08635 & 1.10283 \\
\hline 5 & 1.029502 & 1.06957 & 1.085242 & 1.097385 & 1.04842 & 1.41497 & 1.54166 & 1.58682 \\
\hline 6 & 1.520080 & 1.54341 & 1.527047 & 1.582049 & 1.43423 & 1.83910 & 1.97555 & 2.05464 \\
\hline 7 & 1.997513 & 2.03018 & 2.015643 & 2.024425 & 1.83447 & 2.26557 & 2.41533 & 2.50322 \\
\hline 8 & 2.464020 & 2.48740 & 2.469668 & 2.428527 & 2.24498 & 2.69479 & 2.85427 & 2.93059 \\
\hline
\end{tabular}

TABLE 4. The $L_{2}$ and $L_{\infty}$ critical exponents of several dual refinable functions $\phi_{\left(g_{r}\right)^{s}}^{Q}$ for $r=1, \cdots, 4$ and $k=2, \cdots, 8$ which are constructed by the CBC algorithm in Theorem 5.2.

\begin{tabular}{||c|c|c|c|c|c|c|c|c||}
\hline$k$ & $\nu_{2}\left(\phi_{\left(g_{1}\right)_{k}^{s}}^{Q}\right)$ & $\nu_{2}\left(\phi_{\left(g_{2}\right)_{k}^{s}}^{Q}\right)$ & $\nu_{2}\left(\phi_{\left(g_{3}\right)_{k}^{s}}^{Q}\right)$ & $\nu_{2}\left(\phi_{\left(g_{4}\right)_{k}^{s}}^{Q}\right)$ & $\nu_{\infty}\left(\phi_{\left.\left(g_{1}\right)_{k}^{s}\right)}^{Q}\right.$ & $\nu_{\infty}\left(\phi_{\left.\left(g_{2}\right)_{k}^{s}\right)}^{Q}\right)$ & $\nu_{\infty}\left(\phi_{\left(g_{3}\right)_{k}^{s}}^{Q}\right)$ & $\nu_{\infty}\left(\phi_{\left(g_{4}\right)_{k}^{s}}^{Q}\right)$ \\
2 & 0.31415 & 0.34139 & 0.49278 & 0.58373 & -0.61191 & -0.56260 & -0.39550 & -0.30636 \\
\hline 3 & 0.91646 & 0.93317 & 0.93619 & 1.08366 & 0.048833 & 0.09230 & 0.10659 & 0.26887 \\
\hline 4 & 1.47539 & 1.44569 & 1.48384 & 1.48143 & 0.666544 & 0.65846 & 0.71084 & 0.71639 \\
\hline 5 & 2.00516 & 1.96174 & 1.98167 & 1.99754 & 1.247190 & 1.22045 & 1.25623 & 1.28161 \\
\hline 6 & 2.51561 & 2.44983 & 2.43792 & $\mathrm{~N} / \mathrm{A}$ & 1.798678 & 1.74645 & 1.75268 & 1.80521 \\
\hline 7 & $\mathrm{~N} / \mathrm{A}$ & $\mathrm{N} / \mathrm{A}$ & $\mathrm{N} / \mathrm{A}$ & $\mathrm{N} / \mathrm{A}$ & 2.327900 & 2.26061 & 2.25877 & 2.29371 \\
\hline 8 & $\mathrm{~N} / \mathrm{A}$ & $\mathrm{N} / \mathrm{A}$ & $\mathrm{N} / \mathrm{A}$ & $\mathrm{N} / \mathrm{A}$ & 2.839886 & 2.75635 & 2.74558 & 2.75035 \\
\hline
\end{tabular}




\section{REFERENCES}

[1] A. S. Cavaretta, W. Dahmen, and C. A. Micchelli, Stationary Subdivision, Memoirs of Amer. Math. Soc., 93 (1991). MR 92h:65017

[2] D. R. Chen, B. Han, and S. D. Riemenschneider, Construction of multivariate biorthogonal wavelets with arbitrary vanishing moments, Adv. Comput. Math., 13 (2000), pp. 131-165.

[3] C. K. Chui, J. Stöckler and J. D. Ward, Compactly supported box spline wavelets, Approx. Theory Appl., 8 (1992), 77-100. MR 94a:42042

[4] A. Cohen and I. Daubechies, Non-separable bidimensional wavelet bases, Revista Matemàtica Iberoamericana, 9 (1993), 51-137. MR 94k:42047

[5] A. Cohen, I. Daubechies and J.C. Feauveau, Biorthogonal bases of compactly supported wavelets, Comm. Pure Appl. Math., 45 (1992), 485-560. MR 93e:42044

[6] I. Daubechies, Ten Lectures on Wavelets, SIAM, Philadelphia, (1992). MR 93e:42045

[7] G. Deslauriers and S. Dubuc, Symmetric iterative interpolation processes, Constr. Approx., 5 (1989), 49-68. MR 90c:65016

[8] G. Deslauriers, J. Dubois, and S. Dubuc, Multidimensional iterative interpolation, Can. J. Math., 43 (1991), 297-312. MR 92g:41003

[9] N. Dyn and D. Levin, Interpolating subdivision schemes for the generation of curves and surfaces, in: Multivariate Approximation and Interpolation, W. Haussmann and K. Jetter (eds.), Birkhauser Verlag, Basel, (1990), 91-106. MR 92h:41005

[10] N. Dyn, J. A. Gregory, and D. Levin, A butterfly subdivision scheme for surface interpolation with tension control, ACM Trans. on Graphics, 9 (1990), 160-169.

[11] T. N. T. Goodman, C. A. Micchelli, and J. D. Ward, Spectral radius formulas for subdivision operators, in: Recent Advances in Wavelet Analysis, L. L. Schumaker and G. Webb (eds.), Academic Press, (1994), 335-360. MR 94m:47076

[12] B. Han, On dual wavelet tight frames, Appl. Comput. Harmon. Anal., 4 (1997), 380-413. MR 98h:42031

[13] B. Han, Analysis and construction of optimal multivariate biorthogonal wavelets with compact support, SIAM J. Math. Anal., 31 (2000), 274-304. CMP 2000:08

[14] B. Han and R. Q. Jia, Multivariate refinement equations and convergence of subdivision schemes, SIAM J. Math. Anal., 29 (1998), 1177-1199. MR 99f:41018

[15] B. Han and R. Q. Jia, Optimal interpolatory subdivision schemes in multidimensional spaces, SIAM J. Numer. Anal., 36 (1999), 105-124. MR 99m:65019

[16] H. Ji, S. D. Riemenschneider and Zuowei Shen, Multivariate compactly supported fundamental refinable functions, duals and biorthogonal wavelets, Studies in Appl. Math., 102 (1999), 173-204. MR 99m:42049

[17] R. Q. Jia, Approximation properties of multivariate wavelets, Math. Comp., 67 (1998), 647665. MR 98g:41020

[18] R. Q. Jia, Characterization of smoothness of multivariate refinable functions in Sobolev spaces, Trans. Amer. Math. Soc., 351 (1999), 4089-4112. MR 99m:42050

[19] R. Q. Jia and C. A. Micchelli, On linear independence of integer translates of a finite number of functions, Proc. Edinburgh Math. Soc., 36 (1992), 69-85. MR 94e:41044

[20] R. Q. Jia and S. R. Zhang, Spectral properties of the transition operator associated to a multivariate refinement equation, Linear Algebra Appl., 292 (1999), 155-178. CMP 99:14

[21] J. Kovačević and M. Vetterli, Nonseparable multidimensional perfect reconstruction filter banks and wavelet bases for $\mathbb{R}^{n}$, IEEE Trans. Info. Theory, 38 (1992), 533-555. MR 93b:94002

[22] Y. Meyer, Wavelets and Operators, Vol. 1, Cambridge Press, Cambridge, (1992). MR 94f: 42001

[23] C. A. Micchelli, Interpolatory subdivision schemes and wavelets, J. Approx. Theory, 86 (1996), 41-71. MR 97g:42030

[24] J. P. Mongeau and G. Deslauriers, Continuous and differentiable multidimensional iterative interpolation, Linear Algebra Appl. 180 (1993), 95-120. MR 94e:65017

[25] S. D. Riemenschneider and Zuowei Shen, Multidimensional interpolatory subdivision schemes, SIAM J. Numerical Analysis, 34 (1997), 2357-2381. MR 99j:65011

[26] W. Sweldens, The lifting scheme: a custom-design construction of biorthogonal wavelets, Appl. Comput. Harmon. Anal., 3 (1996), 186-200. MR 97b:42060

[27] L. F. Villemoes, Continuity of nonseparable quincunx wavelets, Appl. Comput. Harmonic Anal., 1 (1994), 180-187. MR 96c:42074 
Program in Applied and Computational Mathematics, Princeton University, PrinceTON, NEW JERSEY 08544

Current address: Department of Mathematical Sciences, University of Alberta, Edmonton, Alberta, Canada T6G 2G1

E-mail address: bhan@math.princeton.edu, bhan@math.ualberta.ca

URL: http://www.math.princeton.edu/ bhan, http://www.math.ualberta.ca/ bhan

Department of Mathematical Sciences, University of Alberta, Edmonton, Alberta, Canada T6G 2G1

E-mail address: jia@xihu.math.ualberta.ca

$U R L:$ http://xihu.math.ualberta.ca/ jia 